\title{
Updating and optimizing error parton distribution function sets in the Hessian approach. II.
}

\author{
Tie-Jiun Hou, ${ }^{1, *}$ Zhite Yu, ${ }^{2, \dagger}$ Sayipjamal Dulat, ${ }^{3, \downarrow}$ Carl Schmidt, ${ }^{2,}$ and C.-P. Yuan ${ }^{2, \|}$ \\ ${ }^{1}$ Department of Physics, College of Sciences, Northeastern University, Shenyang 110819, China \\ ${ }^{2}$ Department of Physics and Astronomy, Michigan State University, East Lansing, Michigan 48824, USA \\ ${ }^{3}$ School of Physics Science and Technology, Xinjiang University, Urumqi, Xinjiang 830046 China
}

(Received 23 August 2019; published 13 December 2019)

\begin{abstract}
In an earlier publication, we introduced the software package Error PDF Updating Method Package (ePump) that can be used to update or optimize a set of parton distribution functions (PDFs), including the best-fit PDF set and Hessian eigenvector pairs of PDF sets (i.e., error PDFs), and to update any other set of observables, in the Hessian approach. Here, we validate the ePump program with a detailed comparison against a full global analysis, and we demonstrate the potential of ePump by presenting selected phenomenological applications relevant to the Large Hadron Collider. For example, we use the package to estimate the impact of the recent LHC data of the measurements of $W$ and $Z$ boson and top quark pair differential distributions on the CT14HERA2 PDFs.
\end{abstract}

DOI: 10.1103/PhysRevD.100.114024

\section{INTRODUCTION}

An understanding of uncertainties due to parton distribution functions (PDFs) is crucial to precision studies of the standard model as well as to searches for new physics beyond the standard model at hadron colliders, such as the CERN Large Hadron Collider. As extensively discussed in Ref. [1], a technique for estimating the impact of new data on the PDFs, without performing a full global analysis, is extremely useful. (See also Refs. [2-4].) For this purpose, we have developed a software package Error PDF Updating Method Package (ePump), which can be used to obtain both the updated best-fit PDF and updated eigenvector PDFs from an earlier global analysis. The package can also directly update the predictions for experimental observables and their PDF uncertainties without requiring the use of the updated PDFs to recalculate the theory predictions. Finally, an alternative use of the package is to optimize a given set of Hessian PDFs for a particular set of observables so that a reduced number of error PDFs can be used, while maintaining the PDF uncertainty on the observables to any desired precision.

\footnotetext{
tjhou@msu.edu

†yuzhite@msu.edu

sdulat@hotmail.com

sschmidt@pa.msu.edu

yuan@pa.msu.edu
}

Published by the American Physical Society under the terms of the Creative Commons Attribution 4.0 International license. Further distribution of this work must maintain attribution to the author(s) and the published article's title, journal citation, and DOI. Funded by SCOAP.
In Ref. [1], some examples were given, comparing the results of ePump with a full global analysis as well as several phenomenological analyses using ePump. In addition, an exercise using ePump was performed in Ref. [5] to show how to assess the potential of precision measurement of triple differential distributions of high-mass (up to sub$\mathrm{TeV}$ ) Drell-Yan pairs to reduce the PDF induced errors in predicting the cross section of an extra $Z^{\prime}$ boson with mass greater than a few $\mathrm{TeV}$ s produced at the LHC. In this work, we provide further checks and more details of the validation of ePump against the full global analysis machinery, and we provide more examples of using ePump to update current PDFs with new LHC data.

In a global analysis of experimental data, the PDFs are defined as a function of a number of fitting parameters, and in turn, the global $\chi^{2}$ and the theoretical prediction for any observable are also functions of the parameters. The crucial approximations used by ePump are these:

(i) The global $\chi^{2}$ is a quadratic function of the parameters around its global minimum.

(ii) All other relevant quantities (including theoretical predictions of new observables used in the update as well as the PDFs themselves) are linear functions of the parameters.

It is these simplifying assumptions that allow ePump to obtain updated best-fit PDFs and error PDFs and to update the predictions and uncertainties for any other observable, in just a few seconds of CPU time. Note that these approximations are the exact same as those used to calculate the PDF uncertainty for any observable in the Hessian method. However, the impact of these approximations must still be considered when interpreting the 
results from ePump. In addition, subtleties in the calculations of PDF uncertainties, such as the use of dynamical tolerances and tier- 2 penalties, could potentially induce further discrepancies between the predictions of ePump vs a full global analysis. Thus, it is useful to validate ePump against a full global analysis in as many distinct applications as possible.

The paper is structured as follows. In Sec. II, we perform the aforementioned validation of ePump. To do this, we start with a base best-fit and error PDF set, obtained from a global analysis using the CT14HERA2 parametrization. The data included are the CT14HERA2 datasets minus some subset of the data. We then use ePump to update the PDFs by adding back the excluded datasets, and we compare with the standard CT14HERA2 PDFs. If the Hessian approximations were exact, we should find that the ePump predictions reproduce exactly the CT14HERA2 PDFs. Thus, we can test how well the approximations work for different classes of datasets. In Ref. [1], results were shown for this exercise in which the jet data were included by ePump. In this paper, we present more details of this check with jet data and also present additional checks with deeply inelastic scattering (DIS) and Drell-Yan data. In each of these cases, we will see that the updated PDFs obtained from ePump are very close to the global-fit results, i.e., CT14HERA2 PDFs in this case. Furthermore, in Sec. II C, we show, as an example, how to use ePump to directly update the theoretical predictions of the Higgs boson production cross section $\sigma(g g \rightarrow h)$ from gluon fusion in the proton-proton collider, including its uncertainties induced by the updated error PDFs.

The speed of ePump makes it very useful to perform analyses to investigate the influence of multiple datasets on the PDFs that otherwise might require many different timeconsuming global fittings. In Sec. III, we demonstrate how to use ePump to quickly identify the experimental datasets that constrain the CT14HERA2 PDFs most stringently. We find that among all of the 33 datasets included in the CT14HERA2 fits, less than half of them is necessary to effectively constrain the CT14HERA2 PDF errors. Detailed information on the impact of those individual datasets to constrain the CT14HERA2 PDFs, such as which parton flavors and at which $x$ values, will also be discussed.

Of course, one of the main uses for a tool such as ePump is to quickly assess the impact of new datasets prior to updating with a full global analysis. In Sec. IV, we provide two detailed examples of this by using ePump to update the CT14HERA2 PDFs with some recent LHC data. First, we examine the impact from the LHC top quark pair $(t \bar{t})$ production data provided by the ATLAS and CMS collaborations. Second, we examine the impact from the ATLAS $7 \mathrm{TeV}$ data on $W$ and $Z$ productions [6]. We find that, while the $t \bar{t}$ data can provide potential constraints on the $g$ PDF, its impact is quite minimal after we have included the inclusive high transverse momentum $\left(p_{T}\right)$ jet production data from the Tevatron and the LHC in the same fit. On the other hand, we find a large impact on the quark PDFs, particularly in the small- $x$ region, when updated by adding the ATLAS $7 \mathrm{TeV} W$ and $Z$ data [6]. This large deviation of the updated PDFs from the original CT14HERA2 PDFs suggests that the ePump result should only be trusted qualitatively in this case, and for quantitative results with this dataset, a full global fit is required. This conclusion is further supported by examining the magnitude of the two measures $\tilde{d}^{0}$ and $d^{0}$, introduced in Ref. [1], which give the distance between the original and updated PDFs in the parameter space, relative to the updated and original errors, respectively. For the ATLAS $7 \mathrm{TeV} W$ and $Z$ data, the value of $\tilde{d}^{0}=1.49$ indicates that the original best-fit PDF was far outside the error band for the updated PDFs, so the new best fit obtained by ePump is more likely to be affected by nonlinearities in the dependence of the observables and the PDFs on the fitting parameters. This, in turn, could produce results that differ from the true global fit.

Finally, concluding remarks are given in Sec. V.

\section{VALIDATION OF ePump USING DATASETS IN CT14HERA2}

The datasets used for PDF global fitting in CT14HERA2 [7] consist of the HERA Run I + II combined data [8], 15 other sets of DIS data, 14 sets of Drell-Yan data, and 4 sets of jet production data, as listed in Table I and II of Ref. [9]. Here, we will take the CT14HERA2 PDFs, including the best-fit and error sets, as the full global fit result to compare against the results of ePump. We shall see how well ePump reproduces the best-fit PDFs and uncertainty of CT14HERA2 for different classes of experimental data. The test goes as follows. First, we perform a full global analysis with the CT14HERA2 parametrization, using all of the CT14HERA2 data except for a particular subset of data. For instance, when we perform the global analysis with the jet data excluded, we obtain a new set of best-fit and error PDFs, called CT14HERA2mJ. We then use ePump to update CT14HERA2mJ by treating the excluded jet data as "new" data, with the updated PDFs called CT14mJeAll. A comparison between the CT14mJeAll and the CT14HERA2 best-fit and error PDFs can then be used to show how well ePump reproduces the full global analysis for this subset of data. Note that, since ePump depends on quadratic and linear approximations, we should not expect perfect agreement. In addition, in the ePump prediction, there are assumptions in how the tier-2 penalties from the new data affect the updated error PDFs and therefore the uncertainties in the updated PDFs. However, as we shall see, the updated best-fit PDFs and their uncertainties from ePump are pretty close to those from the full global analysis.

We shall perform this analysis three times by removing different subsets of data from CT14HERA2: 1) excluding all of the DIS data except the HERA I + II combined 
data (CT14HERA2mD), 2) excluding all Drell-Yan data (CT14HERA2mY), and 3) excluding all Jet data (CT14HERA2mJ). We then add the excluded data back with ePump and compare the updated PDFs with the CT14HERA2 PDFs. To be precise, for the CT14HERA2 parametrization, there are 27 parameters, corresponding to 54 error PDFs. In addition, two gluon extreme sets (i.e., eigen-PDF sets 55 and 56) are introduced via the Lagrangian multiplier method in the CT14HERA2 fit to enlarge the uncertainty in the $g$ PDF in the small- $x$ region. For our CT14HERA2mD, CT14HERA2mY, and CT14HERA2mJ fits, we do not produce these extra gluon extreme sets. Thus, everywhere in this section, we shall exclude the two gluon extreme sets also in the CT14HERA2 PDF errors, in order to have a truer comparison. For convenience, we summarize our notations in this paper here:

(i) CT14HERA2mD, CT14HERA2mY, and CT14HER$\mathrm{A} 2 \mathrm{~mJ}$ are the base sets as described above, to be used by ePump.

(ii) The letter "e" followed by a dataset name indicates that the PDFs are obtained from ePump by adding the given dataset as new data to the base set. For example, in Sec. III A, the PDFs CT14mJeCDF are obtained from ePump by adding the CDF inclusive jet data to the base set CT14HERA2mJ.

(iii) The letters "eAll" indicate that PDFs are obtained from ePump by adding back all of data that were excluded in the base set as new data. Thus, these sets are the ePump approximation to be compared with the full CT14HERA2 PDF set.

(iv) The suffixes ".54" or ".52" (as for example, in CT14HERA2.54) are used to indicate that the error bands are obtained with 54 or 52 eigen-PDFs, respectively, rather than with the full 56 eigen-PDFs.

Finally, we note that we always show symmetric error bands in this paper. As described in Ref. [1], the symmetric Hessian error bands are invariant under a change of the eigen-PDF basis (unlike the asymmetric errors) and therefore are more reliable when assessing the impact of new data on the PDF errors when using ePump.

\section{A. CT14HERA2 excluding all DIS data (except HERA Run I + I I combined): CT14HERA2mD}

There are 3287 data points in total in the CT14HERA2 fit [7,9]. Among these, the DIS experiments contribute 2381 data points, of which 1120 data points are from the precision HERA Run I + II combined neutral current and charged current data. If we remove all of the DIS data from the CT14HERA2 fit, this only leaves 906 data points for the reduced (non-DIS) global fit, with the 2381 DIS data points to be added in by ePump as new data. In this instance, we may not expect ePump to reproduce the full CT14HERA2 fit results well, since there are more data points (2381) in the new data than in the "old" data (906). As a consequence, in many regions of PDF parameter space the new DIS data constrain the PDFs much more than the old non-DIS data. As discussed in Ref. [1], if the updated fit moves too far from the original one in the parameter space, the linear and quadratic approximations used in ePump may break down. Furthermore, it is difficult to obtain a well-converged global fit if all of the DIS data are removed because too many fitting parameters are left unconstrained. If one insists on removing all of the DIS data (including HERA Run I + II), some of the 27 (without extreme sets) or 28 (with extreme sets) parameters must be fixed before doing the fit. Then, one can get a set of global-fit PDFs with fewer parameters than CT14HERA2 and can still use ePump to update them. However, since some parameters that should be constrained by DIS data are already fixed, the update obtained by adding the DIS data using ePump will not fully reflect the impact of the new data, and the comparison with CT14HERA2 becomes less meaningful.

Therefore, in the present analysis, we choose to keep the HERA Run I + II combined data in the original base fit, allowing us to use the full 27 free parameters. These data provide important information on the decomposition of parton flavors inside the proton and therefore provide sufficient constraints on the PDFs for a reasonable base set to update with ePump. Our base fit, CT14HERA2mD, is then obtained from a global fit to a total of 2026 data points, which include all non-DIS data and the HERA I + II combined data and exclude all other DIS data. The remaining DIS data contain 1261 data points, which will be taken as new data to update the CT14HERA2mD PDFs by ePump. The updated PDFs, named CT14mDeAll, can then be compared to the CT14HERA2 PDFs.

In Fig. 1, we compare the ePump-updated PDFs (CT14mDeAll) to the base PDFs (CT14HERA2mD) and the true global-fit PDFs (CT14HERA2). It can be seen that the update with ePump yields results very similar to those of the true global fit. Given the quadratic and linear approximations in ePump, and the number of new data points (1261) compared to the number of old data points (2026), the results are extremely satisfactory. Moreover, these wellapproximated updated PDFs were calculated in just a few seconds of CPU time. So, prior to a full global fit, one can quickly obtain a first look at the impact of the new data using ePump. As is expected, the DIS data provide important information on the $u$ and $d$ PDFs, the error bands of which have shrunk by almost one-half with the inclusion of the non-HERA DIS data. It is also evident that the $u$ quark PDF is constrained more than the $d$ quark PDF. This is easily understood by the fact that the electric charge of the $u$ quark is twice that of the $d$ quark, and so it contributes more to the cross section in low-energy DIS neutral current processes.

\section{B. CT14HERA2 excluding all Drell-Yan data: CT14HERA2mY}

Here, we perform a similar study for the Drell-Yan data in CT14HERA2. The global fit to all the DIS and jet 

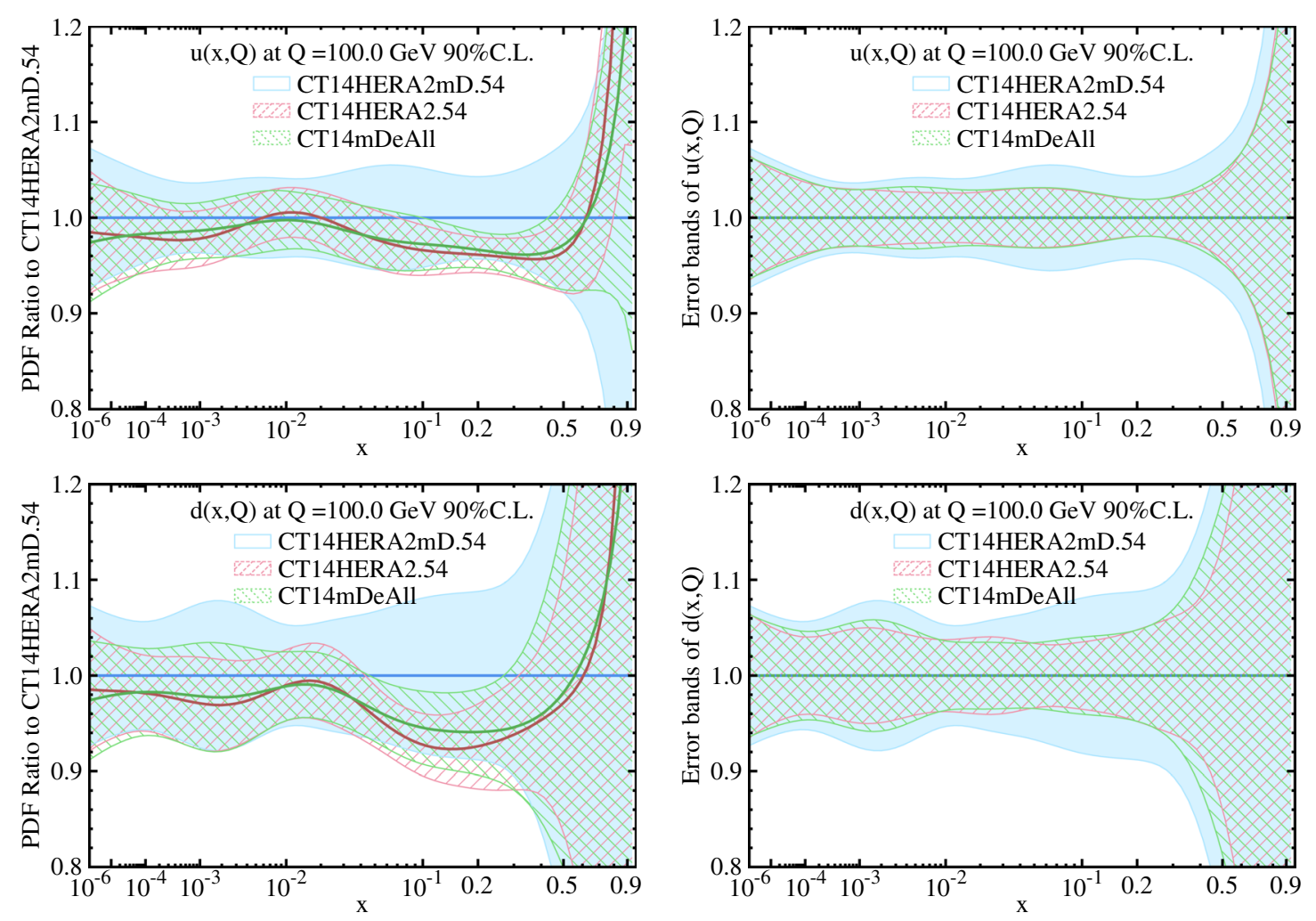

FIG. 1. Comparison of ePump-updated $u$ and $d$ PDFs, at $Q=100 \mathrm{GeV}$. Left panel: the PDF ratios over the best fit of the base CT14HERA2mD. Right panel: the error bands relative to their own best fit.

data, and excluding the Drell-Yan data, is named CT14HERA2mY, which contains 2786 data points. It is worth mentioning that the global fit for CT14HERA2mY with 27 parameters is not very well converged, for the same reason explained in Sec. II A. Fortunately, we only need to fix one parameter to get a good fit. Thus, we are left with enough (26) free parameters to test ePump, and the results are still meaningful, as we will see in the following. The ePump-updated PDFs, obtained by adding back all the Drell-Yan data, which contain 501 data points, to the CT14HERA2mY fit, are shown in Fig. 2, together with CT14HERA2 (after removing the two extreme $g$ PDF sets). Again, we see that ePump yields a result very similar to the true global fit CT14HERA2, with only a small difference for $x$ less than about 0.4 , which is negligible compared to the size of the error band. In the large- $x$ region, the PDFs are small, and there are few experimental data to constrain them, so they are determined by analytic extrapolation and depend strongly on the nonperturbative parametrization forms assumed at the PDF initial scale (which is $1.3 \mathrm{GeV}$ in the CT14HERA2 fit). Therefore, we are not concerned by the differences in the best-fit PDFs at $x$ greater than about 0.4 , which are nevertheless still well within the error bands.

The updated $s$ PDF is shown in Fig. 3, in which one finds a dramatic difference in the $s$ PDF uncertainty between ePump and the global fit. The CT14HERA2 error band of the $s$ PDF increases for almost all $x$ values when the Drell-Yan data is added, while the ePump-updated CT14mYeAll error band stays the same as the base CT14HERA2mY. An increase in the PDF error band found in a global fit usually indicates the presence of some tension between the new and the old data. Because of the quadratic approximation in ePump, it can never produce an increase in the size of the error band, but rather in most cases, it will reduce the error. This can be inferred directly from Eq. (20) in Ref. [1] by the positivity of $\lambda^{(r)}$. Thus, we find that when there is strong tension between the new and old datasets, it will not be revealed by an enlargement of the ePump-updated PDF error bands, in contrast to that of a true global fit. We shall discuss other methods to explore possible tension between different datasets with ePump later.

\section{CT14HERA2 excluding all jet data: CT14HERA2mJ}

CT14HERA2 contains four sets of inclusive jet production data: CDF [10] and D0 [11] at the Tevatron Run-2 and ATLAS $7 \mathrm{TeV}$ [12] and CMS $7 \mathrm{TeV}$ [13] at the LHC. We denote the global fit to all the CT14HERA2 data, minus the four jet datasets, as CT14HERA2mJ, which contains 2882 data points. ${ }^{1}$ The ePump-updated PDFs, obtained by adding

${ }^{1}$ CT14HERA2mJ contains 27 free parameters. We do not need to fix any parameters. 

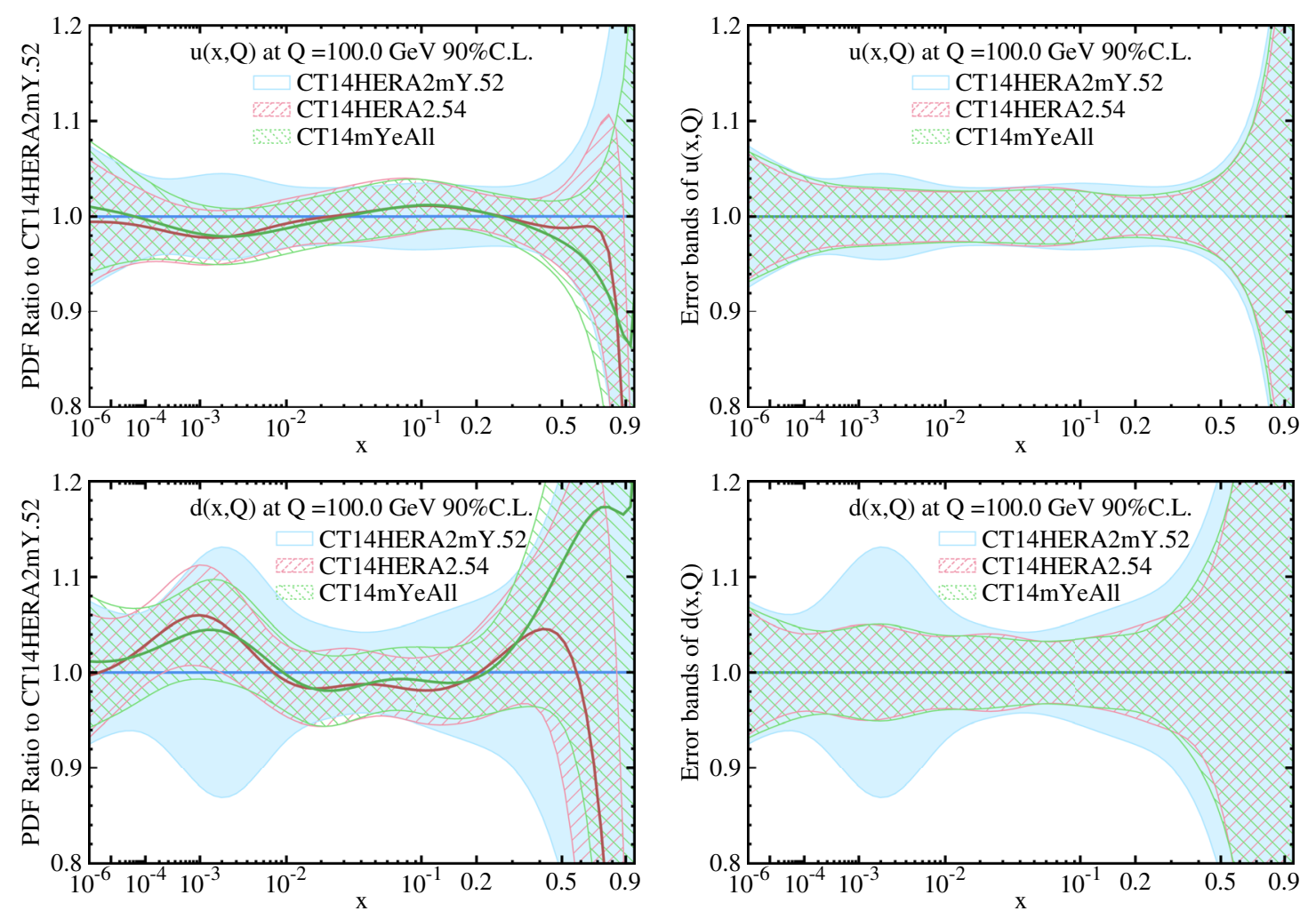

FIG. 2. Comparison of ePump-updated $u$ and $d$ PDFs, at $Q=100 \mathrm{GeV}$. Left panel: the PDF ratios over the best fit of the base CT14HERA2mY. Right panel: the error bands relative to their own best fit.
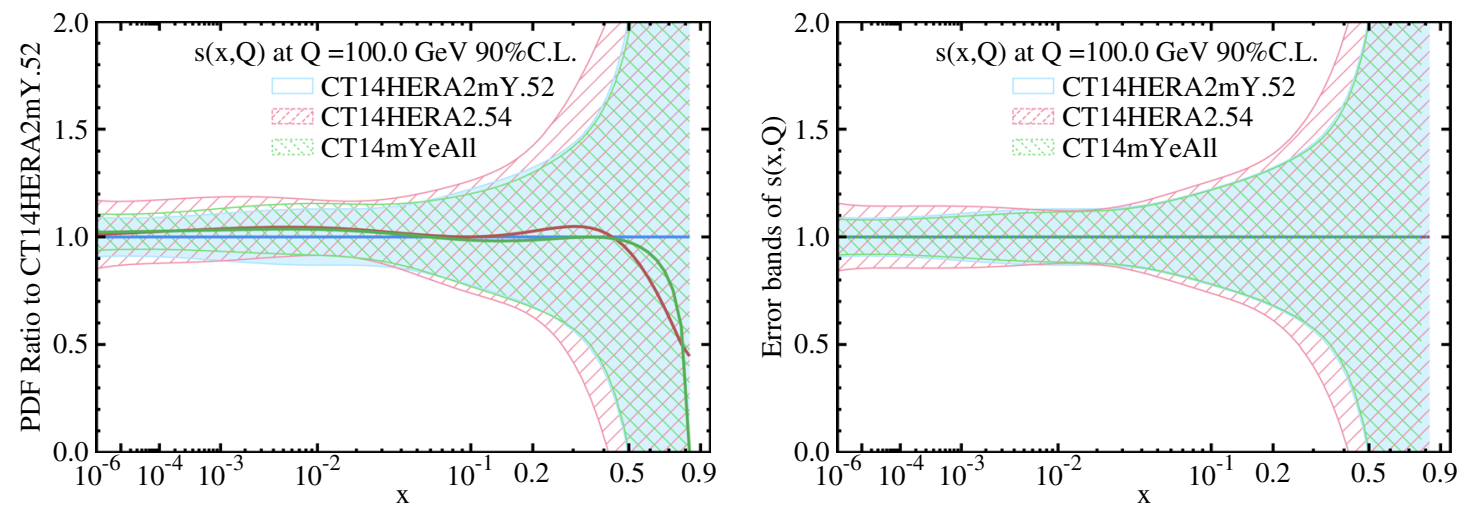

FIG. 3. Same as Fig. 2, but for $s$ PDF.

back the four jet datasets as new data, which contain 405 data points, are designated as CT14mJeAll. As shown in Fig. 4, the jet data mainly constrain the $g$ PDF, with little effect on the $u$ and $d$ PDFs, cf. Fig. 5. The agreement between the ePump-updated PDF and the CT14HERA2 global fit is quite satisfactory. The $g$ PDF is modified, and the error band is increasingly reduced as $x$ grows from 0.01 to 0.3 . At large- $x$ values, the difference in the best-fit PDFs is not significant due to the large error band size. Again, the somewhat larger CT14HERA2 PDF error band of $d$ PDF at $x$ in the range from 0.1 to 0.4 , as compared to CT14mJeAll, indicates some tension caused by adding the jet data to the rest of the CT14HERA2 data in the global fit, which ePump is unable to see.

The Higgs boson cross section is strongly dependent on the $g$ PDF, so an interesting question to ask is what is the impact of the jet data included in the CT14HERA2 fit on the prediction of the Higgs boson production cross section $\sigma(g g \rightarrow h)$ at the LHC. As explained in Sec. II. D of Ref. [1], ePump can update not only the PDFs but also physical observables within a few seconds of CPU time. Table I shows the result of the comparison between the ePump-updated prediction and the CT14HERA2 prediction for various LHC center-of-mass energies. After adding jet 

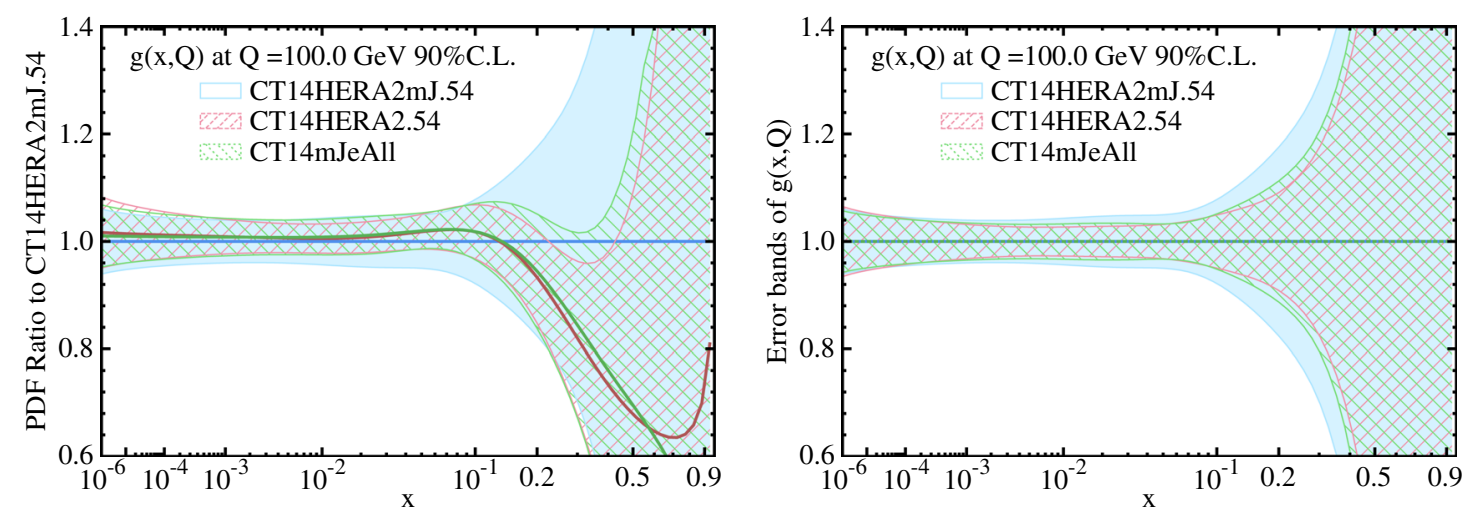

FIG. 4. Comparison of ePump-updated $g$ PDFs, at $Q=100 \mathrm{GeV}$. Left panel: the PDF ratios over the best fit of the base CT14HERA2mJ. Right panel: the error bands relative to their own best fit.
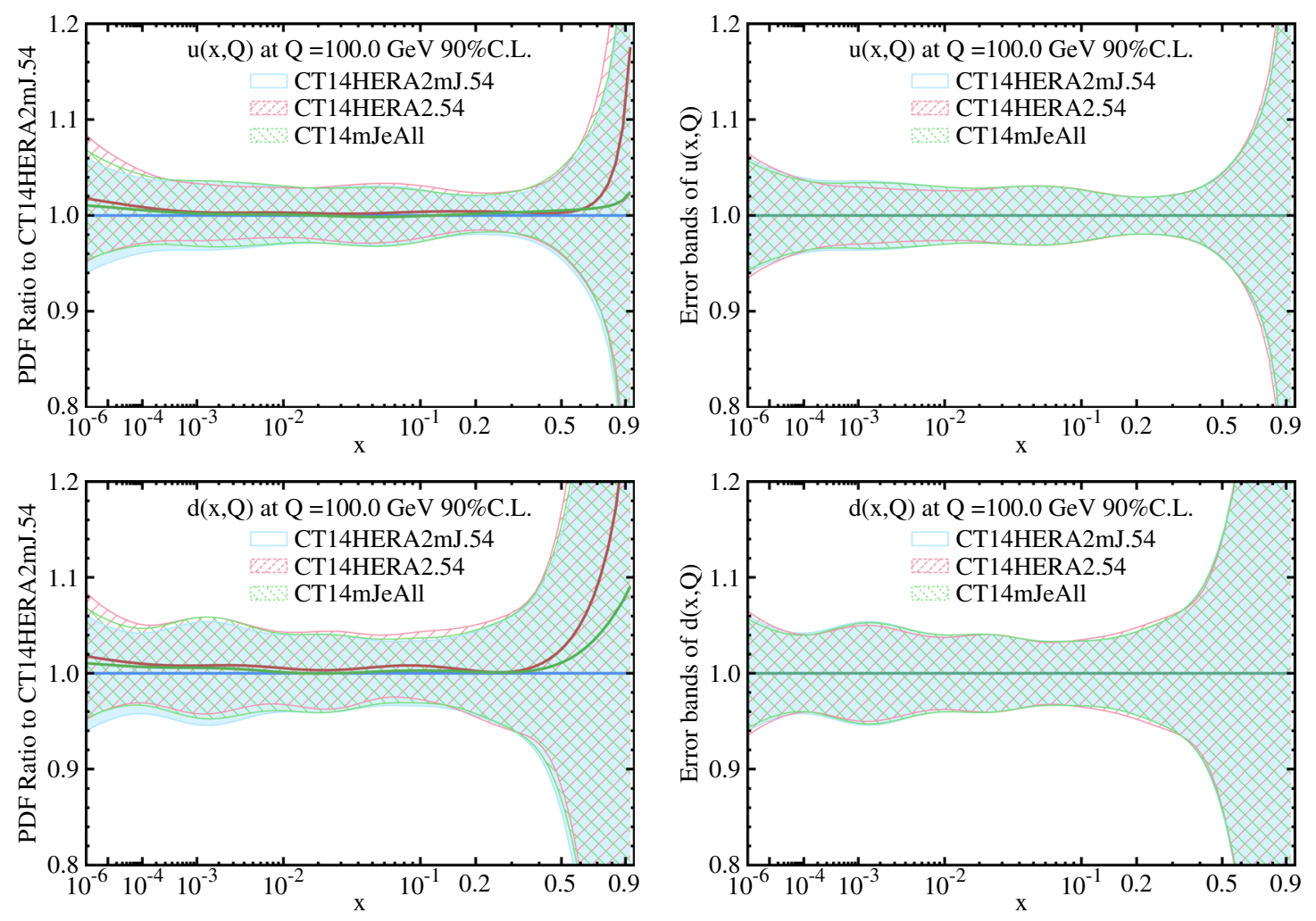

FIG. 5. Same as Fig. 4, but for $u$ and $d$ PDFs.

data into the fits, the central values of $\sigma(g g \rightarrow h)$ become slightly larger, and the errors become smaller. Using ePump, the jet data reduce the error of $\sigma(g g \rightarrow h)$ by about $20 \%$, while the true global fit results in about a $30 \%$ reduction. This difference may be due to the linear and quadratic approximations in ePump, or it could be due to the effect of the new data on the tier-2 penalty, which is only treated on average in ePump. Nevertheless, one can use ePump to quickly estimate the impact of some new data to updated PDFs and physical observables. For instance, in this case, we could conclude from ePump updating that including jet data in the fit will lead to a
TABLE I. Theoretical predictions of $\sigma(g g \rightarrow h)$ in picobarns at the LHC, for various center-of-mass energies, based on different PDF sets. CT14mJeAll predictions were obtained directly from ePump after adding jet data to update the CT14HERA2mJ PDFs. Here, the listed CT14HERA2 PDF errors do not include the contribution from the two extreme $g$ PDF sets.

\begin{tabular}{lccc}
\hline \hline$\sqrt{S}(\mathrm{TeV})$ & CT14HERA2mJ & CT14mJeAll & CT14HERA2 \\
\hline 7 & $14.52 \pm 0.62$ & $14.68 \pm 0.50$ & $14.60 \pm 0.43$ \\
8 & $18.37 \pm 0.80$ & $18.59 \pm 0.63$ & $18.49 \pm 0.53$ \\
13 & $42.1 \pm 2.0$ & $42.7 \pm 1.5$ & $42.5 \pm 1.2$ \\
14 & $47.5 \pm 2.3$ & $48.2 \pm 1.8$ & $48.0 \pm 1.4$ \\
\hline \hline
\end{tabular}


more precise result of $\sigma(g g \rightarrow h)$ with its uncertainty reduced by about $20 \%$.

\section{IMPACT OF INDIVIDUAL CT14HERA2 DATASETS ON PDFs}

ePump can be used to quickly assess the impact of individual datasets on constraining the PDFs in a global analysis. In this section, we will demonstrate this by using ePump to assess the datasets used in the CT14HERA2 global analysis.

\section{A. Impact of jet data in the CT14HERA2 fit}

As already noted in Sec. II C, the jet data mainly constrain the $g$ PDF and have little effect on other flavors. From Fig. 4, we see that the jet data prefer a larger $g$ PDF at $x=10^{-2} \sim 10^{-1}$ and smaller $g$ PDF at $x=0.2 \sim 0.4$. The error band is reduced by a fairly large amount in the range of $x=10^{-2} \sim 0.2$, by about $1 / 4$ to $1 / 3$.

To see the impact of individual jet data in the CT14HERA2 fit, we use ePump to add each jet dataset individually to CT14HERA2mJ. The results are shown in Fig. 6, with CT14mJeAll shown together in the same graph for comparison. It can be seen that the four jet datasets produce the same qualitative effects on the $g$ PDF, but quantitatively the CMS $7 \mathrm{TeV}$ jet data [13] yield the result
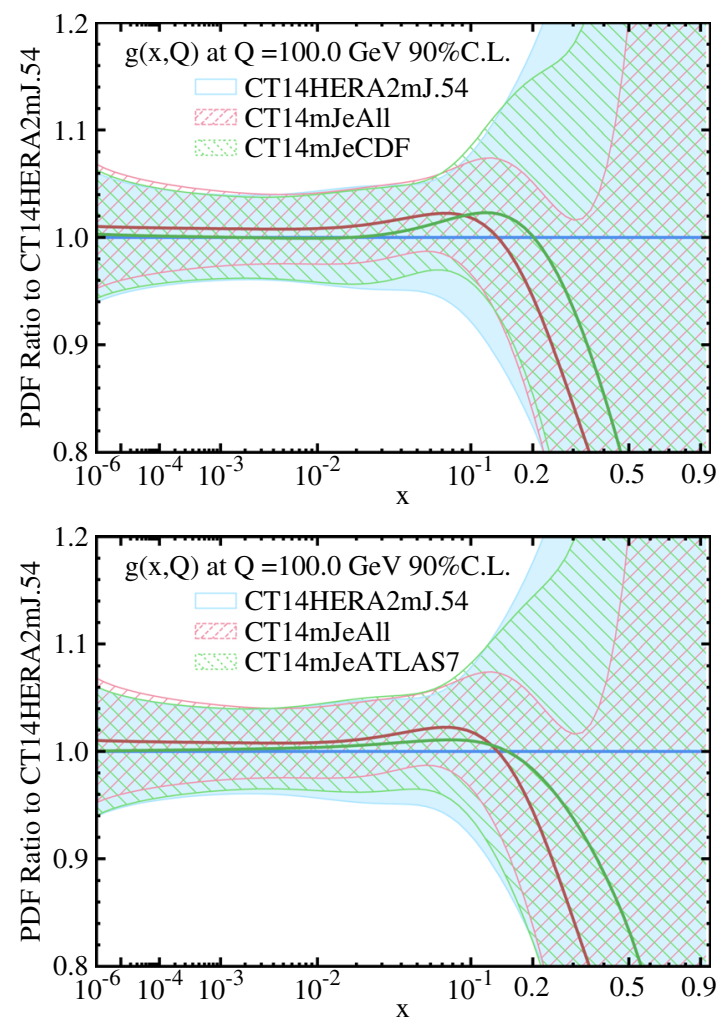

that is most similar to CT14mJeAll. It increases the $g$ PDF slightly at small $x$, with maximum pull upward around $x \sim 0.1$, but pulls it downward sharply above $x \sim 0.2$. While all of the jet datasets reduce the error band, the CMS $7 \mathrm{TeV}$ jet data reduce it the most and are the closest to the all-jet result. The others reduce the errors by a distinctly smaller amount. From this, we can draw the conclusion that the CMS jet data have the dominant impact on the $g$ PDF, among all the jet data included in CT14HERA2. It is worth noting that in the range of $x=0.1 \sim 0.2$ the CDF Run-2 inclusive jet dataset leads to a harder $g$ PDF than the others, while the D0 Run-2, ATLAS $7 \mathrm{TeV}$ and CMS $7 \mathrm{TeV}$ jet data yield similar results for the $g$ PDF.

\section{B. Impact of Drell-Yan data in the CT14HERA2 fit}

The impact of Drell-Yan data on $u, d$, and $s$ PDFs has been shown in Figs. 2 and 3 in Sec. II B. The impact on other flavor PDFs can be found in Figs. 7 and 8. It is found that Drell-Yan data mainly constrain $u, d, \bar{u}$ and $\bar{d}$ PDFs, with little effect on $g$ and $s$ PDFs. Drell-Yan data not only reduce the uncertainties of $u, d, \bar{u}$, and $\bar{d}$ a lot but also change the best-fit PDFs dramatically. It is worth noting that for most values of $x$ the Drell-Yan data pull the $u$ and $d$ PDFs in opposite directions, cf. Fig. 2. Around $x=10^{-3}$, the $u \mathrm{PDF}$ is decreased, while the $d \mathrm{PDF}$ is increased, and at $x \sim 0.1, u$ becomes larger, while $d$ becomes smaller.
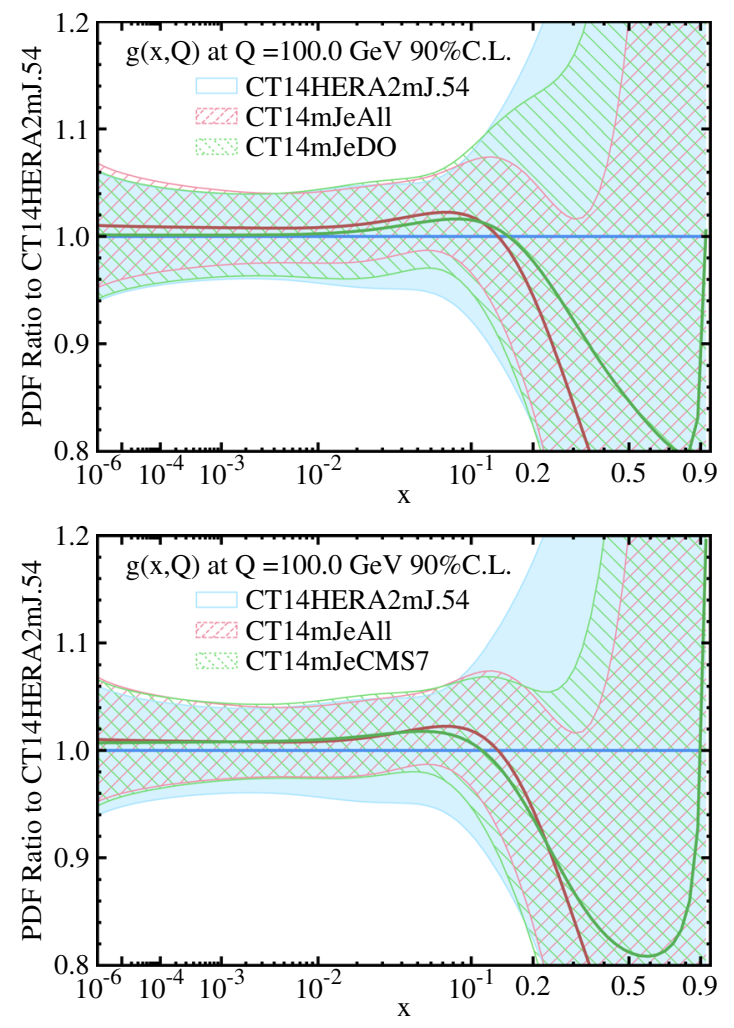

FIG. 6. Impact of individual jet datasets on the $g$ PDF, using ePump to add the datasets to CT14HERA2mJ, which are then compared with CT14mJeAll. The curves are the ratios to the best fit of CT14HERA2mJ, and they can be used to identify the relative impact of each jet dataset on the $g$ PDF. 

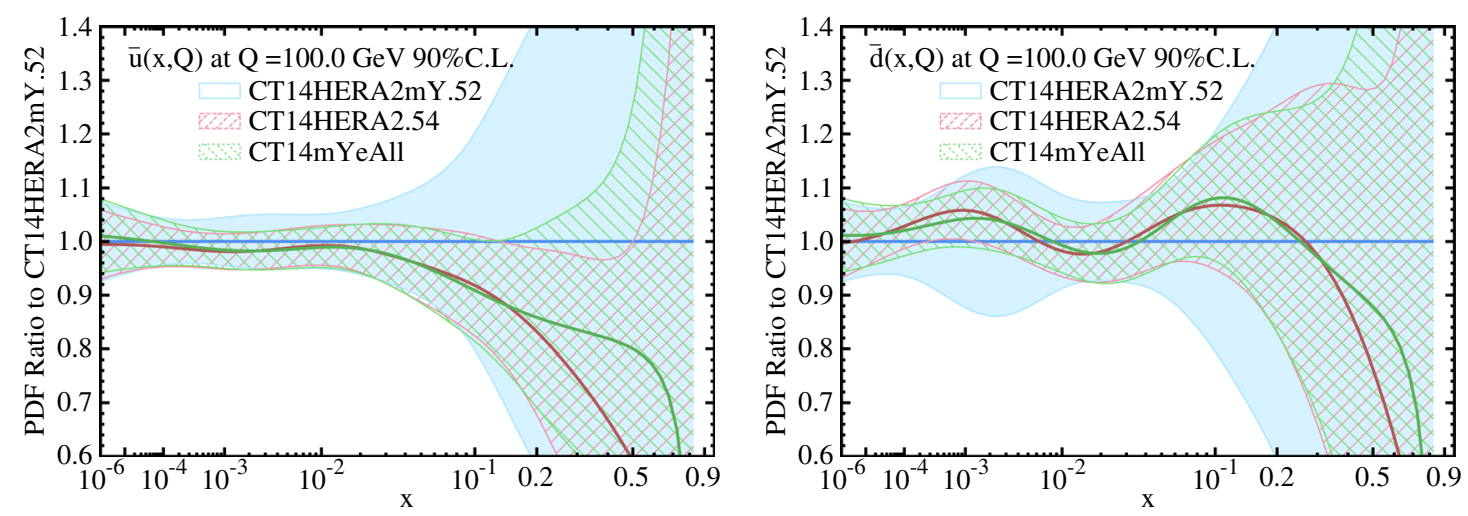

FIG. 7. Same as Fig. 2, but for $\bar{u}$ and $\bar{d}$ PDFs.
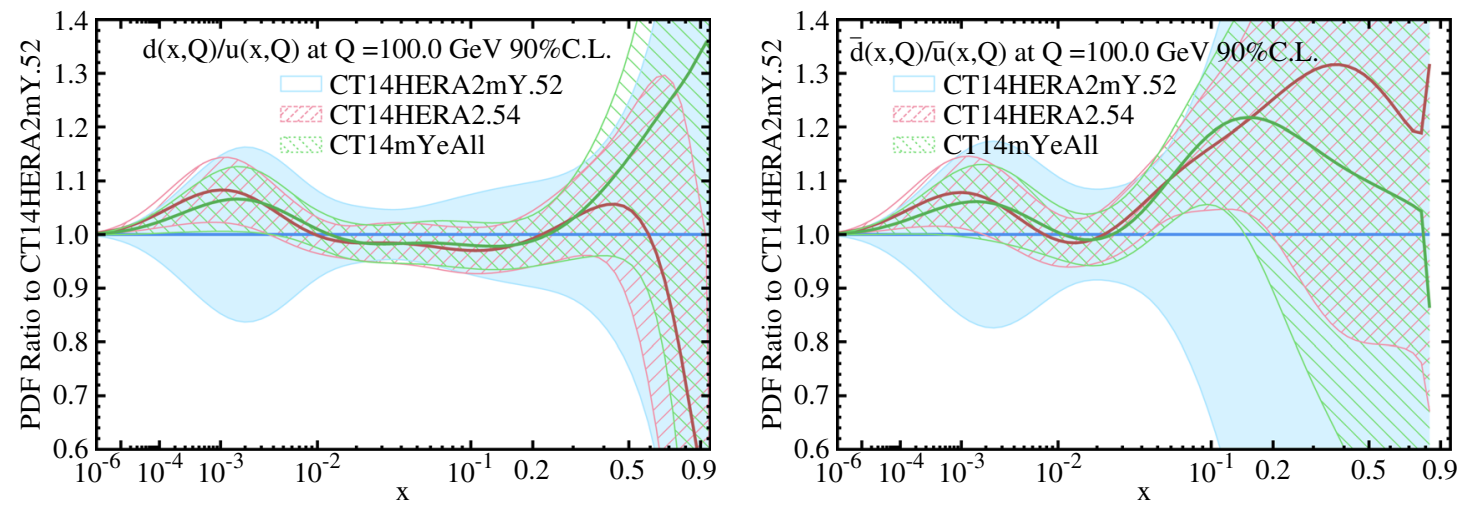

FIG. 8. Same as Fig. 2, but for $d / u$ and $\bar{d} / \bar{u}$ PDF ratios.

This feature is also visible for the $\bar{u}$ and $\bar{d}$ PDFs and is a characteristic of Drell-Yan data.

The fact that the $u$ and $d$ PDFs or the $\bar{u}$ and $\bar{d}$ PDFs get pulled in opposite directions indicates that the fitting program is directly modifying the differences between them. Namely, it is $(u-d)$ and $(\bar{u}-\bar{d})$, or rather the ratios $d / u$ and $\bar{d} / \bar{u}$, that are directly probed by the Drell-Yan data. A reasonable conjecture is that this is due to $W^{ \pm}$charge asymmetry data measured at the Tevatron and the LHC. To check this, we use ePump to add each Drell-Yan dataset individually to CT14HERA2mY and compare with CT14mYeAll, which we have already shown is very close to CT14HERA2. We find that, although most datasets give a similar trend, only the $\mathrm{CMS} 7 \mathrm{TeV} \mu$ asymmetry data [14], the CMS $7 \mathrm{TeV}$ electron asymmetry data [15], the ATLAS $7 \mathrm{TeV} W Z$ data [6], and the D0 Run $2 \mu$ asymmetry

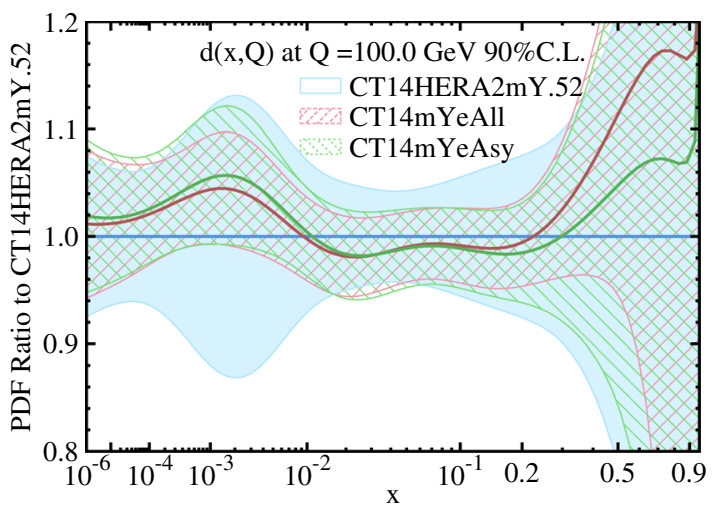

FIG. 9. Comparison of CT14mYeAsy and CT14mYeAll for $u$ and $d$ PDFs at $Q=100 \mathrm{GeV}$. CT14mYeAsy is obtained by adding CMS $7 \mathrm{TeV} \mu$ asymmetry data, CMS $7 \mathrm{TeV}$ electron asymmetry data, ATLAS $7 \mathrm{TeV} W Z$ data, and D0 Run $2 \mu$ asymmetry data to CT14HERA2mY, using ePump. The PDF ratios are over the best fit of CT14HERA2mY. 

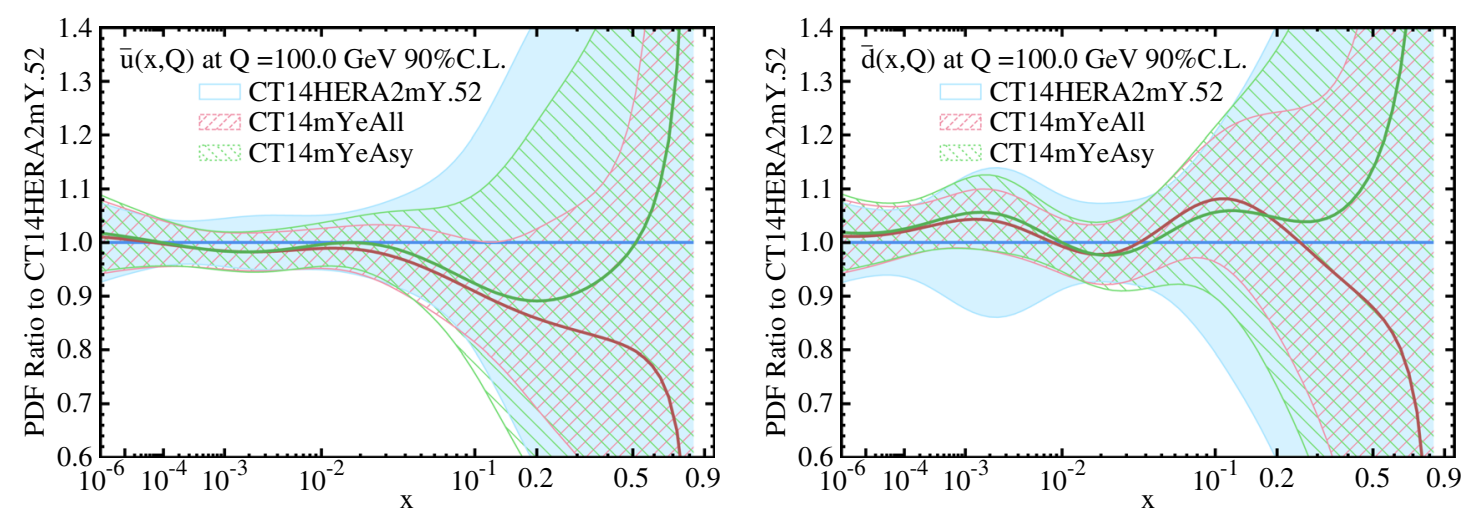

FIG. 10. Same as Fig. 9, but for $\bar{u}$ and $\bar{d}$ PDFs.

data [16] have an appreciable impact. This result is as expected, since most of them are lepton charge asymmetry data. We can use ePump to add just these four charge asymmetry datasets to CT14HERA2mY. The result, called CT14mYeAsy, is shown in Figs. 9-11, compared with CT14mYeAll. The results from just including the lepton charge asymmetry data are pretty close except in the large- $x$ region, where the relative PDF errors are large and the approximations used by ePump are unreliable [1].

Another important Drell-Yan dataset is the E866 data [17], which measure the ratio of Drell-Yan production in proton-deuteron and proton-hydrogen collisions, $\sigma(p d) /$ $2 \sigma(p p)$. These data impose important constraints on the PDF ratios $\bar{d} / \bar{u}$ and $d_{v} / u_{v}$ at larger values of $x$. Using ePump to update CT14HERA2mY PDFs by taking the E866 data as new data, we obtained the CT14mYeE866 PDFs, which are compared to the CT14HERA2 PDFs in Fig. 12. This figure shows that at $x=0.02 \sim 0.2$ the antiquark PDF ratios, $\bar{d} / \bar{u}$, and the valence quark ratios, $d_{v} / u_{v}$, and their error bands are greatly constrained and are very close to the CT14mYeAll fit. Comparing Figs. 10-12, we conclude that both the lepton charge asymmetry data (from the Tevatron and the LHC) and the E866 data are needed to closely reproduce the CT14HERA2 fit result for the $\bar{u}, \bar{d}, \bar{d} / \bar{u}$, and $d_{v} / u_{v}$ PDFs.

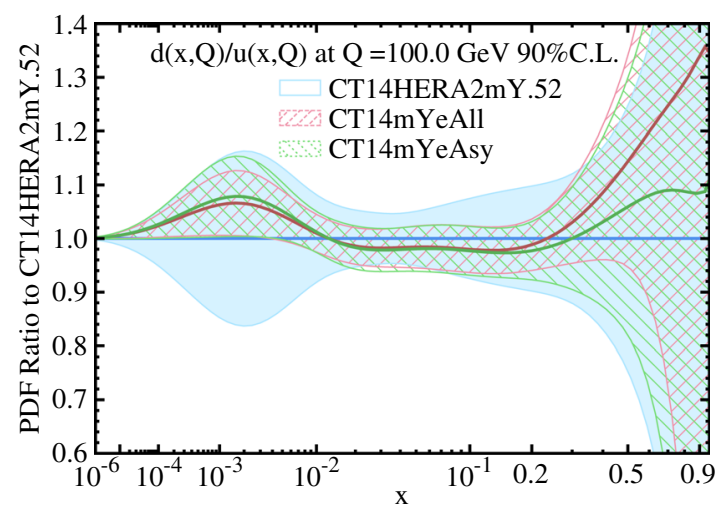

\section{Impact of DIS data in the CT14HERA2 fit}

The DIS datasets provide important information on the $u$ and $d$ PDFs, even when the HERA run I + II combined data have already been included, as shown in Fig. 1. In contrast to the Drell-Yan case, the DIS data pull the $u$ and $d$ PDFs in the same direction. This feature also holds for the $\bar{u}$ and $\bar{d}$ PDFs, as shown in Fig. 13. The implication is that precision DIS data are more sensitive to the sum (or, rather, the weighted sum) than the difference or ratio between the $d$ and $u$ PDFs (or $\bar{d}$ and $\bar{u}$ PDFs). This is because most of the precision DIS data measured the $F_{2}$ structure function. Only a few experiments provided precision measurements of the $F_{3}$ structure function, which probes the difference between $u$ and $d$ or $\bar{u}$ and $\bar{d}$. In Fig. 14, we show the impact of DIS data (excluding HERA I + II) on $d / u$ and $\bar{d} / \bar{u}$ in the CT14HERA2 fit, which is seen to be relatively small.

The impact of DIS data on the $g$ PDF is shown in Fig. 15. It is interesting to note that the DIS data prefer a harder gluon in the $x>0.2$ region. This is opposite of the effect of the jet data, which prefer a softer gluon at $x>0.2$, cf. Fig. 4. The tension between these two kinds of data on the $g$ PDF can be seen by noting that the error band of the true global fit CT14HERA2 is wider than that obtained by the ePump updating, cf. Fig. 15.

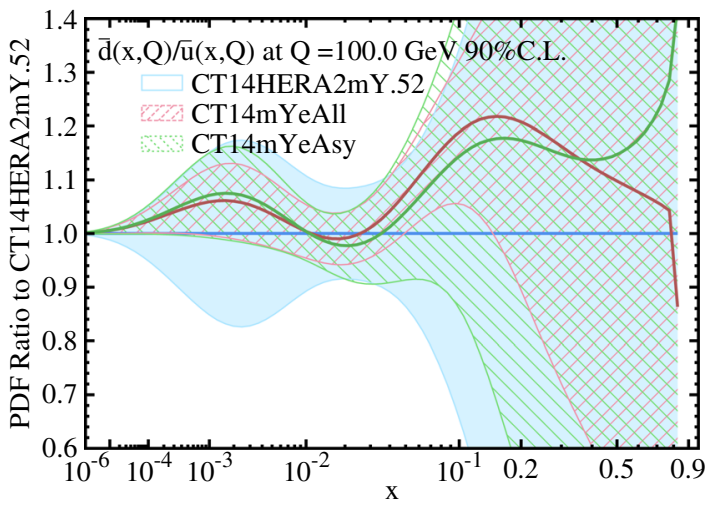

FIG. 11. Same as Fig. 9, but for $d / u$ and $\bar{d} / \bar{u}$ PDF ratios. 

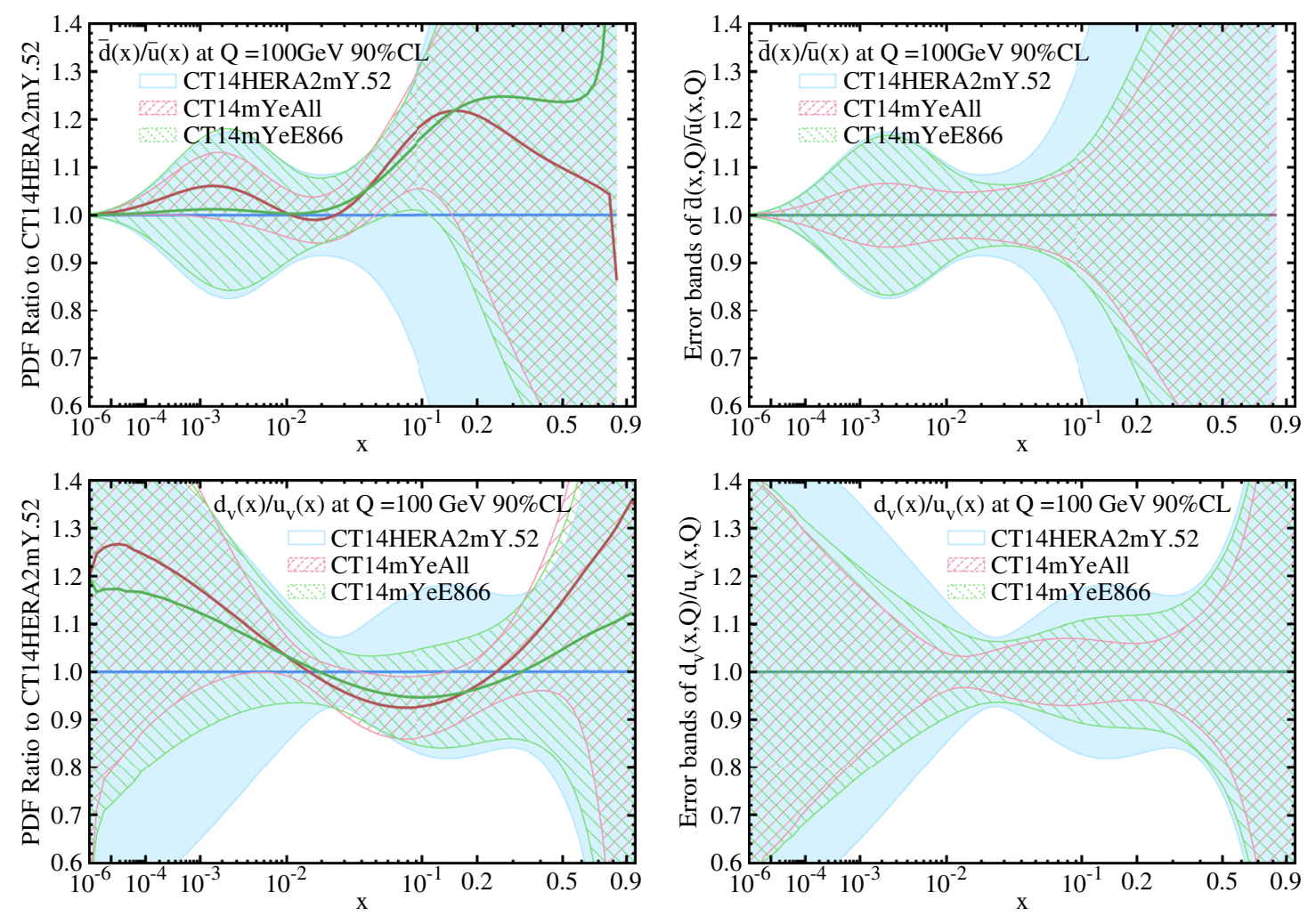

FIG. 12. Updated PDF ratios $\bar{d} / \bar{u}$ and $d_{v} / u_{v}$ with their error bands, at $Q=100 \mathrm{GeV}$, when using ePump to add E866 data to CT14HERA2mY. Left panel: the PDF ratios for ePump-updated CT14mYeE866 and CT14mYeAll over the best fit of the base CT14HERA2mY. Right side: the error bands relative to their own best fit.

DIS data are also expected to constrain the strange quark $(s)$ PDF. For most of the DIS data, however, the contribution to the $s$ PDF is much smaller than to the $u$ and $d$ PDFs, except for the DIS charged current dimuon production experiments $[18,19]$, where the strange quark gives the dominant contribution. Thus, it is interesting to see how much the dimuon data alone can constrain the $s$ PDF among all the DIS data. This can be quickly investigated with ePump. In Fig. 16, we compare the updated $s$ PDF, by including only these dimuon data, to the one with the full DIS data included. Although dimuon datasets are

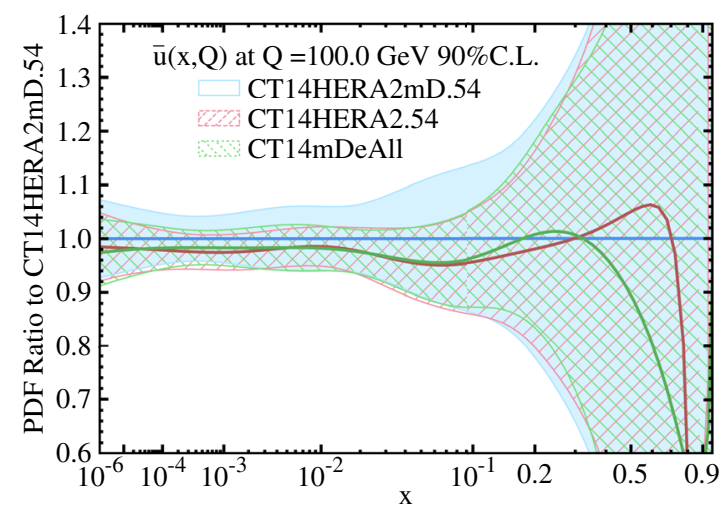

not the only ones responsible for determining the $s$ PDF, they do fully constrain the $s$ PDF over a wide $x$ region, from $10^{-4}$ to $10^{-1}$, for both the central PDF and its error band. Furthermore, we will see that the effects mostly come from $\mathrm{NuTeV}$ dimuon data [18] and only a little from CCFR dimuon data [19].

As before, we can use the ePump updating code to investigate the effect from the individual DIS dataset on the CT14HERA2 PDFs. We find the bulk of the DIS data contributions comes from CCFR $F_{2}^{p}$ [20], $x F_{3}^{p}$ [21], CDHSW $F_{2}^{p}$ and $F_{3}^{p}$ [22], NuTeV $\bar{\nu} \mu \mu$ SIDIS, and

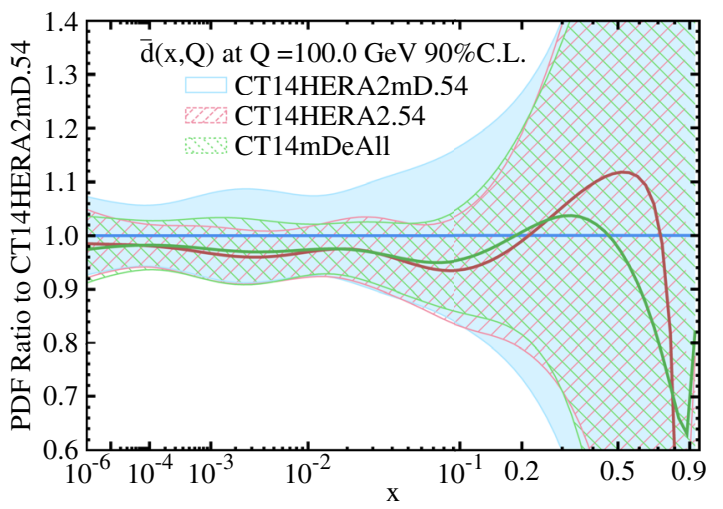

FIG. 13. Same as Fig. 1, but for $\bar{u}$ and $\bar{d}$ quark PDFs. 

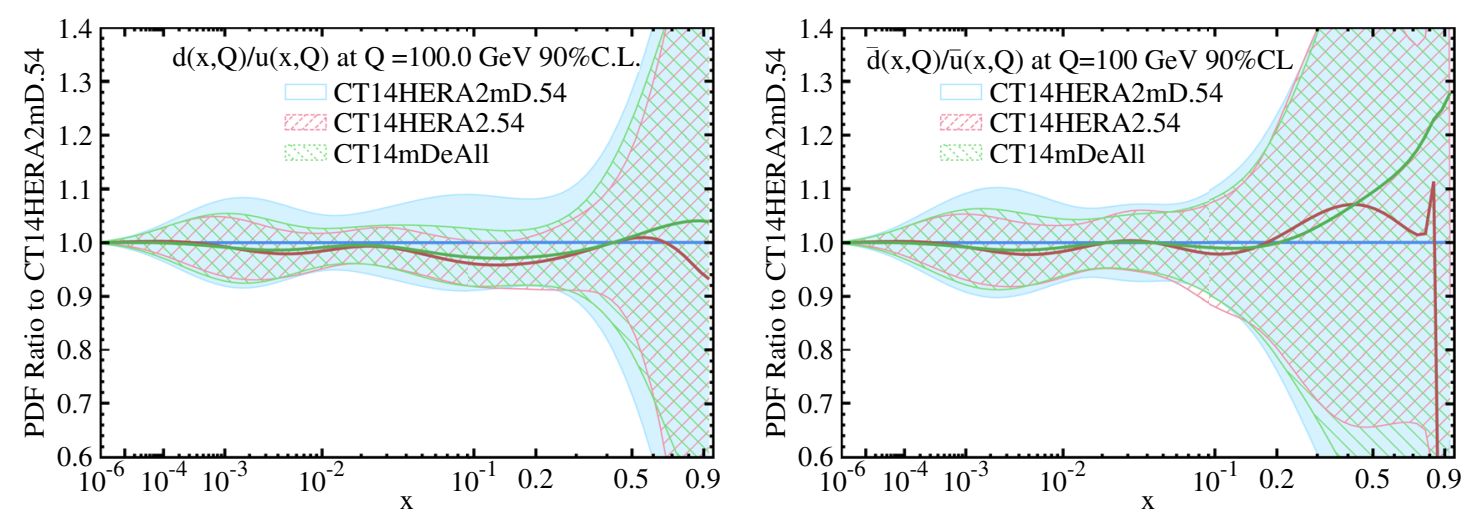

FIG. 14. Same as Fig. 1, but for $d / u$ and $\bar{d} / \bar{u}$ PDF ratios.
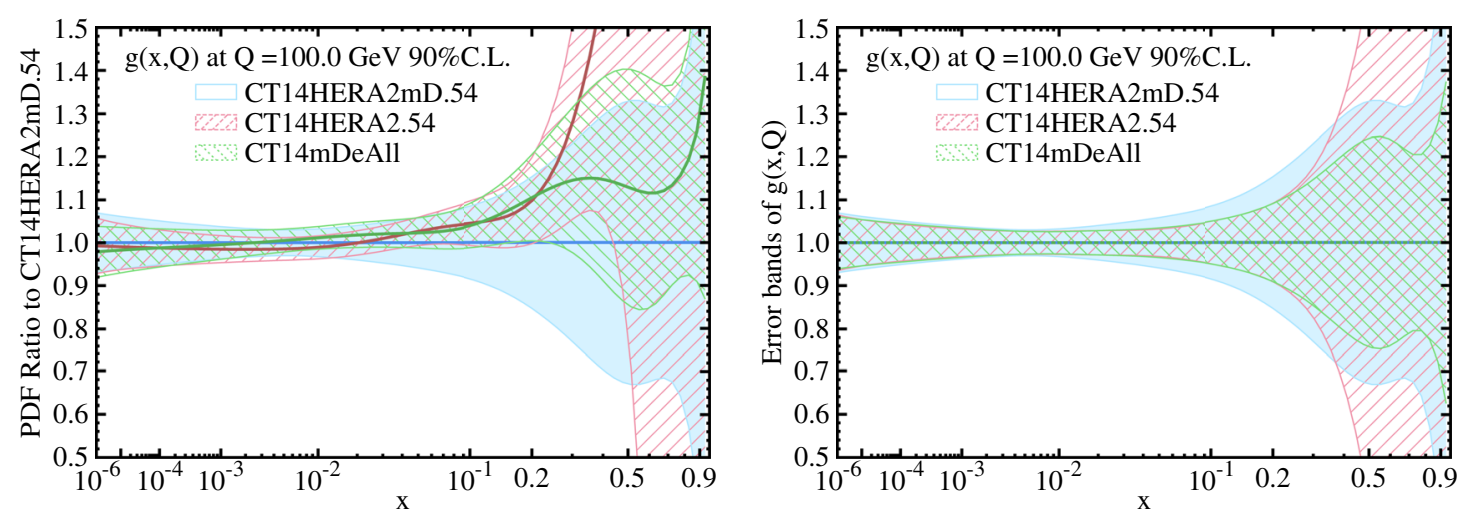

FIG. 15. Same as Fig. 1, but for $g$ PDF.

NuTeV $\nu \mu \mu$ SIDIS [18] datasets. As discussed above, $\mathrm{NuTeV}$ dimuon data are almost solely responsible for constraints on the $s$ PDF, with the stronger constraint coming from NuTeV $\bar{\nu} \mu \mu$ SIDIS data. CCFR and CDHSW $F_{2}^{p}$ data produce the biggest changes to $u$ and $d$, and together with CCFR and CDHSW $F_{3}^{p}$, they give the biggest change to $\bar{u}$ and $\bar{d}$. However, the error band on $d / u$ is not reduced until we also add in $\mathrm{NMC} F_{2}^{d} / F_{2}^{p}$ data [23]. Although the other DIS data do not have as large of an impact as the seven above-mentioned datasets, they are still responsible for some "fine structure" of the PDFs. For example, BCDMS $F_{2}^{p}[24]$ and $F_{2}^{d}[25]$ data measured the structure functions $F_{2}$ of protons and deuterons and cover the large- $x$ region $x \lesssim 0.8$. Hence, these two datasets constrain $u_{v}$ and $d_{v}$ quarks and $g$ PDFs in the large- $x$ region. Because of limited space, we shall not show all the corresponding plots in this paper but instead will post them on the website of the ePump project [26]. For completeness,

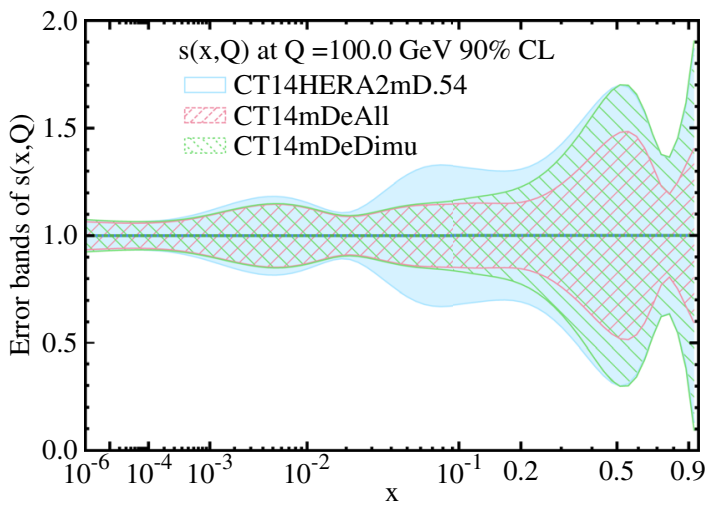

FIG. 16. Comparison of ePump-updated $s$ PDF, at $Q=100 \mathrm{GeV}$. CT14mDeDimu is obtained by adding only the DIS charged current dimuon data (NuTeV [18] and CCFR [19]) to CT14HERA2mD with ePump. 
TABLE II. Impact of individual DIS datasets in CT14HERA2 on the PDFs. For each dataset, only its most prominent effects are listed. The base PDF set used for this study is CT14HERA2mD. Therefore, the effects refer to the net impact when each individual dataset is added, one at a time, to CT14HERA2mD.

\begin{tabular}{llll}
\hline \hline ID & \multicolumn{1}{c}{ Experimental dataset } & & \multicolumn{1}{c}{ Most prominent effects } \\
\hline 101 & BCDMS $F_{2}^{p}$ & {$[24]$} & Reduce $g$ and $u_{v}$ uncertainties at $x: 0.01 \sim 0.7$ \\
102 & BCDMS $F_{2}^{d}$ & {$[25]$} & Reduce $g$ uncertainty at $x: 0.01 \sim 0.7$ and $u_{v}, d_{v}$ at $x \sim 0.2$ \\
104 & NMC $F_{2}^{d} / F_{2}^{p}$ & {$[23]$} & Reduce $d / u, \bar{d} / \bar{u}$, and $d_{v} / u_{v}$ uncertainties for all $x$ \\
108 & CDHSW $F_{2}^{p}$ & {$[22]$} & Almost all the PDFs \\
109 & CDHSW $F_{3}^{p}$ & {$[22]$} & $u_{v}$ and $d_{v}$ central fits for all $x$ \\
110 & CCFR $F_{2}^{p}$ & {$[20]$} & Almost all the PDFs \\
111 & CCFR $x F_{3}^{p}$ & {$[21]$} & $u_{v}$ and $d_{v}$ central fits for all $x$ \\
124 & NuTeV $\nu \mu \mu$ SIDIS & {$[18]$} & $s$ PDF at $x: 0.01 \sim 0.4$ \\
125 & NuTeV $\bar{\nu} \mu \mu$ SIDIS & {$[18]$} & $s$ PDF at $x: 0.01 \sim 0.4$ \\
126 & CCFR $\nu \mu \mu$ SIDIS & {$[19]$} & Reduce $s$ PDF uncertainty at $x \sim 0.1$ \\
127 & CCFR $\bar{\nu} \mu \mu$ SIDIS & {$[19]$} & Reduce $s$ PDF uncertainty slightly at $x \sim 0.1$ \\
145 & H1 $\sigma_{r}^{b}$ & {$[27]$} & Not much effect \\
147 & Combined HERA charm production & {$[28]$} & Not much effect \\
169 & H1 $F_{L}$ & {$[29]$} & Not much effect \\
\hline \hline
\end{tabular}

we summarize our findings in Tables II-IV, in which we list the most prominent effects of each dataset in CT14HERA2. We note that such a study by ePump must start from a base set of global-fit PDFs, and the effects of the data listed in the tables refer to their "net" impact when added, one at a time, to the particular base PDF set. For example, for the DIS data in Table II, the base PDF set is CT14HERA2mD, which includes the Drell-Yan and jet data and the HERA Run I + II data [8]. Thus, it may happen that the effects of some DIS data are similar to those of the HERA Run I + II data, with the result that an individual dataset, such as
H1 $\sigma_{r}^{b}$ data [27], appears to have little or no impact to the updated central set PDFs.

Finally, before leaving this section, we would like to investigate the impact of the CDHSW $F_{2}$ and $F_{3}$ data in the CT14HERA2 fit. It has long been argued that these datasets were not analyzed properly and therefore should not be used in a global fit [38]. Therefore, one may wonder how the CT14HERA2 PDFs would change if we exclude these two CDHSW datasets from the original CT14HERA2 fit. Instead of redoing the whole global fit, we use ePump to quickly answer this question. The procedure is to use ePump

TABLE III. Same as Table II, showing experimental datasets on Drell-Yan processes. The base PDF set for this study is CT14HERA2mY. Therefore, the effects refer to the net impact when each individual dataset is added, one at a time, to CT14HERA2mY.

\begin{tabular}{|c|c|c|c|}
\hline ID & Experimental dataset & & Most prominent effects \\
\hline 201 & E605 Drell-Yan process & {$[30]$} & Pull down $\bar{u}$ and $\bar{d}$ PDFs at $x \gtrsim 0.1$ \\
\hline 203 & E866 Drell-Yan process, $\sigma_{p d} /\left(2 \sigma_{p p}\right)$ & {$[17]$} & $\bar{d} / \bar{u}$ and $d_{v} / u_{v}$ at $x: 0.01 \sim 0.2$ \\
\hline 204 & E866 Drell-Yan process, $Q^{3} d^{2} \sigma_{p p} /\left(d Q d x_{F}\right)$ & {$[31]$} & $\bar{u}$ at $x \gtrsim 0.04$ and $u_{v}$ at $x: 10^{-3} \sim 0.4$ \\
\hline 225 & CDF Run-1 electron $A_{c h}$ & [32] & Reduce errors of $d / u, \bar{d} / \bar{u}$ at $x \sim 0.1$ and $d_{v} / u_{v}$ at all $\mathrm{x}$ \\
\hline 227 & CDF Run-2 electron $A_{c h}$ & [33] & Reduce $d_{v} / u_{v}$ by a little at $x \lesssim 0.3$ \\
\hline 234 & D0 Run-2 muon $A_{c h}$ & [16] & $\begin{array}{l}\text { Reduce } d / u, \bar{d} / \bar{u}, \text { and } d_{v} / u_{v} \\
\text { uncertainties at } x: 10^{-3} \sim 0.3\end{array}$ \\
\hline 240 & $\mathrm{LHCb} 7 \mathrm{TeV} 35 \mathrm{pb}^{-1} W / Z d \sigma / d y_{\ell}$ & [34] & Not much effect \\
\hline 241 & $\mathrm{LHCb} 7 \mathrm{TeV} 35 \mathrm{pb}^{-1} A_{c h}$ & [34] & $\begin{array}{l}\text { Reduce } d / u, \bar{d} / \bar{u}, d_{v} / u_{v} \text { uncertainties } \\
\text { slightly at } x: 10^{-4} \sim 10^{-2}\end{array}$ \\
\hline 260 & D0 Run-2 $Z$ rapidity & {$[35]$} & Not much effect \\
\hline 261 & CDF Run-2 $Z$ rapidity & [36] & Not much effect \\
\hline 266 & CMS $7 \mathrm{TeV} 4.7 \mathrm{fb}^{-1}$, muon $A_{c h} \mathrm{GeV}$ & [14] & Almost all the quark PDFs \\
\hline 267 & CMS $7 \mathrm{TeV} 840 \mathrm{pb}^{-1}$, electron $A_{c h} \mathrm{GeV}$ & {$[15]$} & Almost all the quark PDFs \\
\hline 268 & ATLAS $7 \mathrm{TeV} 35 \mathrm{pb}^{-1} \mathrm{~W} / Z$ cross section, $A_{c h}$ & {$[6]$} & Almost all the quark PDFs \\
\hline 281 & D0 Run-2 $9.7 \mathrm{fb}^{-1}$ electron $A_{c h}$ & [37] & $\begin{array}{l}\text { Reduce } d / u, \bar{d} / \bar{u} \text {, and } d_{v} / u_{v} \\
\text { uncertainties at } x: 10^{-4} \sim 0.3\end{array}$ \\
\hline
\end{tabular}


TABLE IV. Same as Table II, showing experimental datasets on inclusive jet production. The base PDF set for this study is CT14HERA2mJ. Therefore, the effects refer to the net impact when each individual dataset is added, one at a time, to CT14HERA2mJ.

\begin{tabular}{|c|c|c|c|}
\hline ID & Experimental dataset & & Most prominent effects \\
\hline 504 & CDF Run-2 inclusive jet production & {$[10]$} & $g$ PDF at $x: 0.02 \sim 0.5$ \\
\hline 514 & D0 Run-2 inclusive jet production & [11] & $g$ PDF at $x: 0.02 \sim 0.5$ \\
\hline 535 & ATLAS $7 \mathrm{TeV} 35 \mathrm{pb}^{-1}$ inclusive jet production & {$[12]$} & $g$ PDF at $x: 0.02 \sim 0.5$ \\
\hline 538 & CMS $7 \mathrm{TeV} 5 \mathrm{fb}^{-1}$ inclusive jet production & {$[13]$} & $g$ PDF at $x: 0.02 \sim 0.5$ \\
\hline
\end{tabular}

to update the CT14HERA2mD PDFs by taking all but the CDHSW DIS data as new data. The resulting PDFs, CT14eNoCDHSW, are shown in Fig. 17 to be compared with CT14mDeAll, in which all DIS data were included.
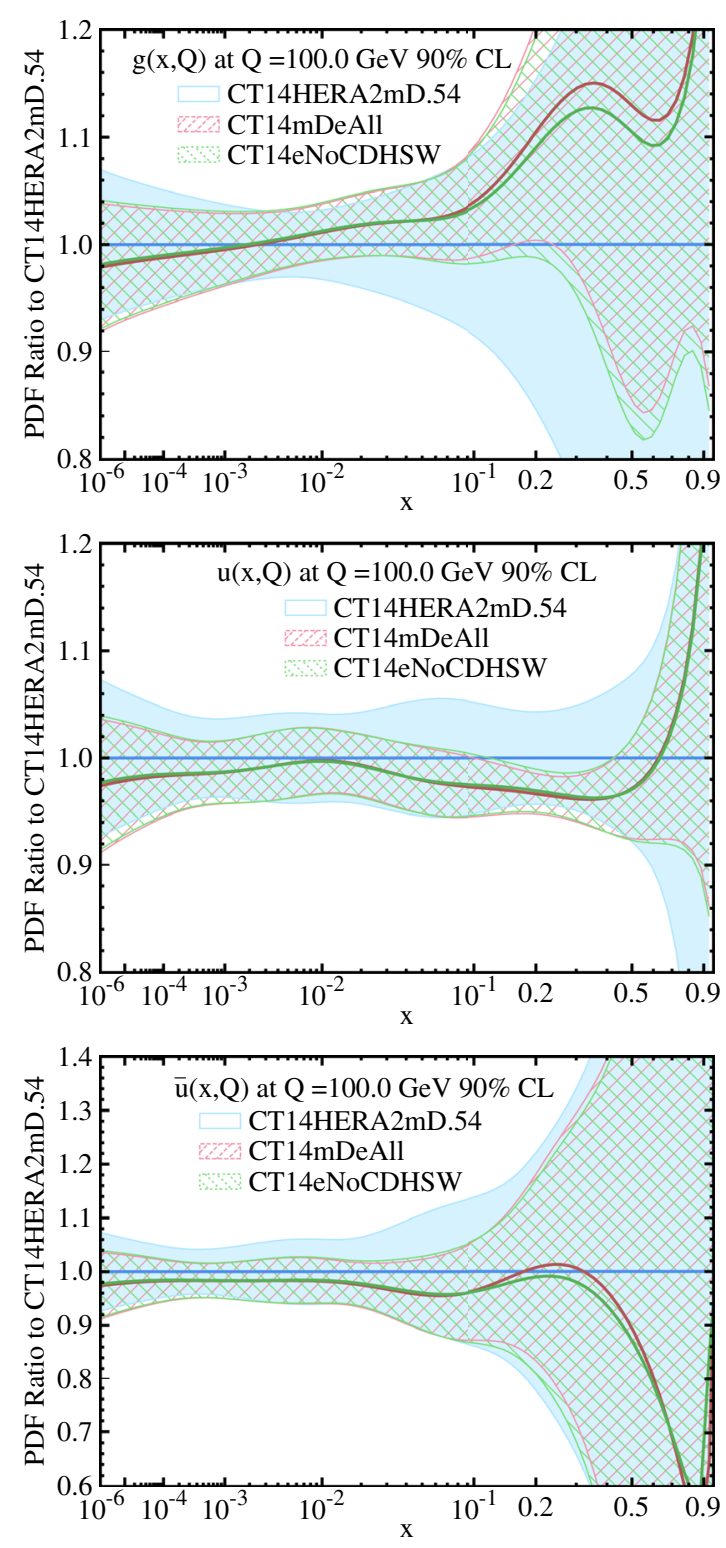

From these figures, we immediately note that they do not differ very much. This is because the CCFR $F_{2}$ and $F_{3}$ data have effects similar to the CDHSW data on CT14HERA2 PDFs. Furthermore, from Fig. 17, we can extract the
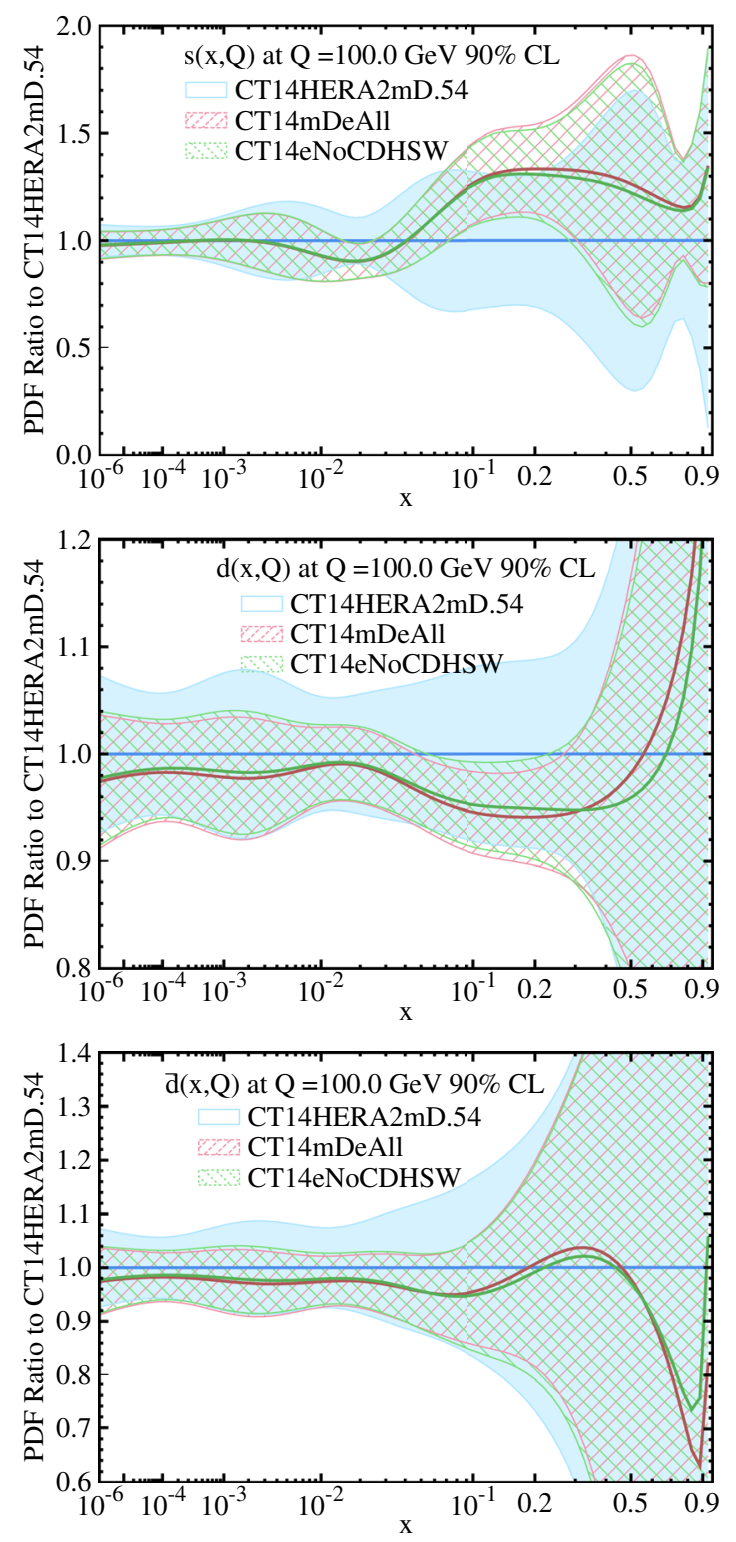

FIG. 17. Comparison of ePump-updated PDFs, at $Q=100 \mathrm{GeV}$. CT14eNoCDHSW is obtained by taking all but the CDHSW DIS data as new data and CT14HERA2 $\mathrm{mD}$ as the base. 
TABLE V. List of $t \bar{t}$ datasets from CMS [40] and ALTAS [41]. $d^{0}$ is the length of shift of the best-fit point in parameter space, as explained in the text. The third column is $d^{0}$ values for each $t \bar{t}$ dataset when added to CT14HERA2 with ePump, and the fourth column is when added to CT14HERA2mJ.

\begin{tabular}{lccc}
\hline \hline ID & Data & $d^{0}$ of CT14HERA2 & $d^{0}$ of CT14HERA2mJ \\
\hline 561 & CMS 8 TeV normalized $d \sigma / \sigma d p_{T}^{t}$ & 0.14 & 0.27 \\
562 & CMS 8 TeV normalized $d \sigma / \sigma d y_{t}$ & 0.06 & 0.23 \\
563 & CMS 8 TeV normalized $d \sigma / \sigma d m_{t \bar{t}}$ & 0.17 & 0.32 \\
564 & CMS 8 TeV normalized $d \sigma / \sigma d y_{t \bar{t}}$ & 0.25 & 0.64 \\
565 & ATLAS 8 TeV absolute $d \sigma / d p_{T}^{t}$ & 0.01 & 0.02 \\
566 & ATLAS 8 TeV absolute $d \sigma / d\left|y_{t}\right|$ & 0.09 & 0.31 \\
567 & ATLAS 8 TeV absolute $d \sigma / d m_{t \bar{t}}$ & 0.03 & 0.01 \\
568 & ATLAS 8 TeV absolute $d \sigma / d\left|y_{t \bar{t}}\right|$ & 0.17 & 0.47 \\
\hline \hline
\end{tabular}

consequences of removing the CDHSW data from the CT14HERA2 fit. Removing the CDHSW data leads to slightly softer $g, s, \bar{u}$, and $\bar{d}$ PDFs at $x>0.1$, and harder $d$ and $u$ PDFs at $x>0.03$. Recall that the Tevatron and LHC jet data also prefer a softer $g$ PDF at $x>0.2$, cf. Fig. 6 . Thus, removing the CDHSW data is more consistent with jet data.

\section{USING ePump TO STUDY THE IMPACT OF NEW DATA}

In the previous sections, we have validated ePump against the CT14HERA2 global fit by updating the PDFs with some subset of the CT14HERA2 datasets. We have also used ePump to investigate the impact of individual datasets on the CT14HERA2 PDFs. In this section, we will use ePump to study the potential impact of some new LHC data on improving the CT14HERA2 PDFs. An example was already given in Ref. [1], in which we analyzed the impact of the CMS inclusive jet production data at $\sqrt{S}=8 \mathrm{TeV}$ [39]. Here, we consider two more examples of the new LHC data: the LHC $8 \mathrm{TeV} t \bar{t}$ differential cross section data and the ATLAS $7 \mathrm{TeV} W^{ \pm}$and $Z$ data.

\section{A. $t \bar{t}$ data at the LHC}

We shall consider eight $t \bar{t}$ datasets presented by the CMS [40] and ALTAS [41] collaborations, as listed in Table V. They are the absolute and normalized onedimensional differential cross sections of the transverse momentum $\left(p_{T}^{t}\right)$ and rapidity $\left(y_{t}\right)$ of top quark and invariant mass $\left(m_{t \bar{t}}\right)$ and rapidity $\left(y_{t \bar{t}}\right)$ of the $t \bar{t}$ pair. The dominant production of $t \bar{t}$ pairs at the LHC is through the gluongluon fusion process. Thus, $t \bar{t}$ data can potentially constrain the $g$ PDF, especially at large values of $x$, due to the large $t \bar{t}$ invariant mass. We also display in the third column of Table $\mathrm{V}$ the measure $d^{0}$, introduced in Ref. [1], which summarizes in a single value the change in the best-fit PDFs after the new data have been added to the original CT14HERA2 fit. To be precise, $d^{0}$ is the length of the shift of the best-fit point in parameter space, relative to the
90\% confidence level (C.L.) boundary of the original PDFs. Thus, $d^{0}=1$ means that the new best fit touches the $90 \%$ C.L. boundary, while a value of $d^{0} \lesssim 0.1$ implies a very small change to the best-fit PDFs. ${ }^{2}$ From Table V, we can see that most of the new $t \bar{t}$ data have a minimal effect on the best-fit CT14HERA2 PDFs, with the CMS $8 \mathrm{TeV}$ normalized $d \sigma / \sigma d y_{t \bar{t}}$ dataset having the biggest impact. The $g$ PDF, updated for this dataset, is shown in Fig. 18. One can see that the updated best-fit $g$ PDF slightly decreases at $x>0.2$, with slightly reduced error band at $x \sim 0.3$, as compared to the CT14HERA2 PDFs. Hence, this dataset prefers a softer $g$ PDF in the large- $x$ region.

Given the dependence of the $t \bar{t}$ distributions on the $g$ PDF, it is interesting to compare the impact of each of the new LHC $t \bar{t}$ datasets with that of the four jet datasets already included in the CT14HERA2 fit. To do this, we use ePump to add each of the individual $t \bar{t}$ datasets as new data to update the CT14HERA2mJ PDFs. Recall that the CT14HERA2mJ PDFs, introduced in Sec. IIC, were obtained from a global fit to all of the CT14HERA2 datasets, except the four Tevatron and LHC jet data. The resulting $d^{0}$ values, after updating from CT14HERA2mJ, are listed in the fourth column of Table V. Comparing to the comparable values in the third column, obtained from updating CT14HERA2, we find that the new $t \bar{t}$ datasets have a much larger effect in the absence of the jet data. In this case, the CMS $8 \mathrm{TeV}$ normalized $d \sigma / \sigma d y_{t \bar{t}}$ and ATLAS $8 \mathrm{TeV}$ absolute $d \sigma / d\left|y_{\bar{t} \bar{t}}\right|$ data have the largest impact, for which the updated $g$ PDFs are shown in Fig. 19. It can be seen that the $y_{t \bar{t}}$ distributions measured at both CMS and ATLAS have comparable effects, and they modify the $g$ PDF similarly to that of the jet data. But the $t \bar{t}$ data have less power to reduce the uncertainties of $g$ PDFs, especially in the $x$ range $0.1 \sim 0.2$. This is consistent with our finding that in the presence of jet data the new $t \bar{t}$ datasets have little effect on PDFs because the $t \bar{t}$ data produce the same change

\footnotetext{
${ }^{2}$ One should note that $d^{0}$ only reflects the change in the best-fit PDFs, so that it is still possible for the new data to produce a significant reduction in the PDF error bands, even if $d^{0}$ is small.
} 

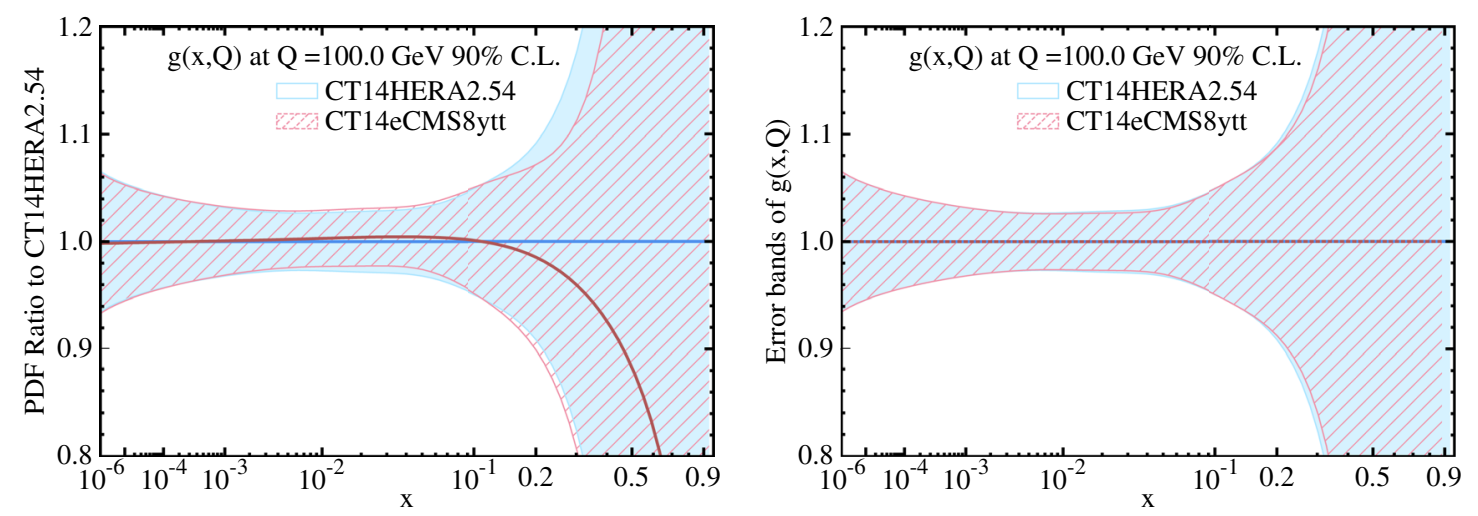

FIG. 18. ePump-updated $g$ PDF, when the CMS $8 \mathrm{TeV}$ normalized $d \sigma / \sigma d y_{t \bar{t}}$ dataset is added to CT14HERA2. Left panel: PDF ratios to the CT14HERA2 best fit. Right panel: the error bands relative to their own best fit.

on the central $g$ PDFs but provide less constraining power on the error band. The reason for this can be traced to the simple fact that there are far fewer $t \bar{t}$ data points than jet data points, due to a smaller production cross section. Thus, the statistical power of $t \bar{t}$ is smaller.

We can test this interpretation using ePump by increasing the weight of the $t \bar{t}$ data in the ePump updating. A weight larger than 1 is equivalent to having more $t \bar{t}$ data points with the same experimental uncertainties or, alternatively, to reducing the experiment uncertainties by a factor of the square root of the weight. Of course, increasing the weight is not exactly the same as increasing the luminosity, since it does not change the central values of the data, which presumably have fluctuations described by the original experimental uncertainties. Nevertheless, one can get some estimate of the potential impact of the $t \bar{t}$ data as the integrated luminosity is increased. To compare with the effect of the jet data, we multiply the contribution of the
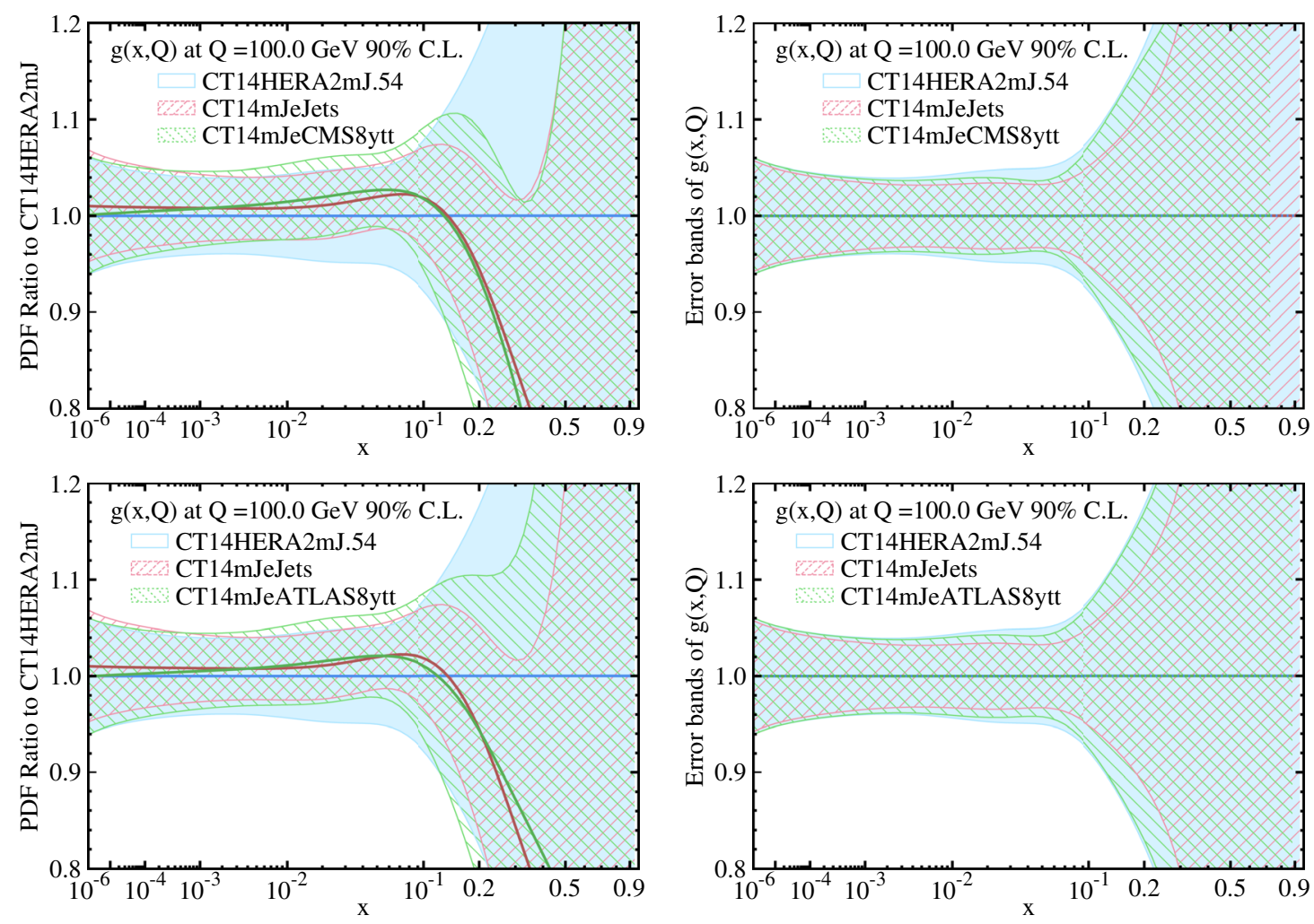

FIG. 19. Updated $g$ PDFs when CMS $8 \mathrm{TeV}$ normalized $d \sigma / \sigma d y_{t \bar{t}}$ data or ATLAS $8 \mathrm{TeV}$ absolute $d \sigma / \sigma d y_{t \bar{t}}$ data are added to CT14HERA2mJ. For comparison, we also display the updated $g$ PDF (CT14HERA2mJeJets), obtained when the jet data in CT14HERA2 are added to CT14HERA2mJ by ePump. Left panel: PDF ratios to the CT14HERA2mJ best fit. Right panel: the error bands relative to their own best fit. 

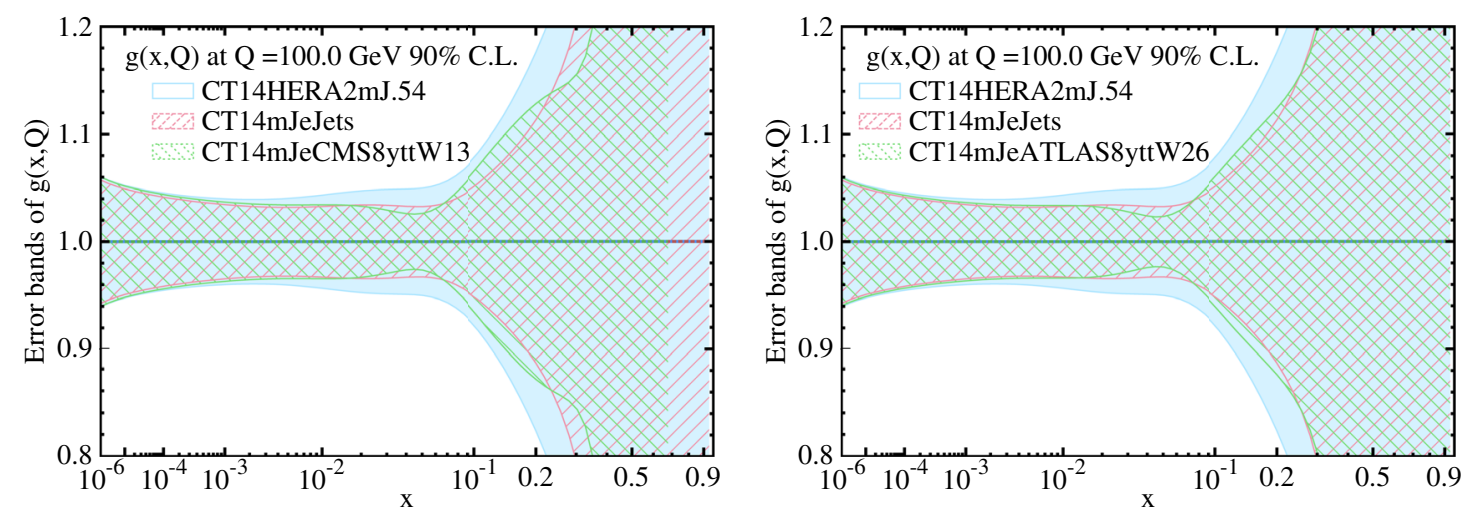

FIG. 20. Same as Fig. 19, with weights 13 and 26 for the CMS $8 \mathrm{TeV}$ normalized $d \sigma / \sigma d y_{t \bar{t}}$ data and ATLAS 8 TeV absolute $d \sigma / \sigma d y_{t \bar{t}}$ data, respectively. Only the error bands are shown in this figure.

new $t \bar{t}$ dataset to $\chi^{2}$ by a weight equal to the ratio of the number of jet data points to the number of individual $t \bar{t}$ data points. We have seen in Sec. III A that the CMS $7 \mathrm{TeV}$ jet data [13], with 133 data points, have the dominant effect among all the jet data in CT14HERA2, so we multiply by the weights $133 / 10=13$ for the CMS and $133 / 5=26$ for the ATLAS $y_{t \bar{t}}$ distributions. The $g$ PDFs, obtained by updating the CT14HERA2mJ fit with the weighted $y_{i \bar{t}}$ distributions using ePump, are shown in Fig. 20. The general shapes of the updated $g$ PDFs are similar to that obtained by including all four jet data in the CT14HERA2 fit. However, the error band of the $g$ PDFs is not reduced as much as the CT14HERA2 fit for $x>0.01$. Hence, we conclude that the jet data will probably impose a stronger constraint on the $g$ PDF than the $t \bar{t}$ data, even with more integrated luminosity collected at a higher center-of-mass energy of the LHC.

Before leaving this section, we comment on the impact of the ATLAS $8 \mathrm{TeV} d \sigma / d p_{T}^{t}$ and $d \sigma / d m_{t \bar{t}}$ data. Given the small values of $d^{0}$ in Table $\mathrm{V}$, we expect little change in the best-fit PDFs when only including these data to update either the CT14HERA2 or CT14HERA2mJ PDFs. This can happen when the theory prediction is in good agreement with the data even before updating. Figure 21 shows that this is indeed the case for CT14HERA2, and similar results were found for CT14HERA2mJ. This is also demonstrated by the small $\chi^{2}$ per data point for these two datasets in Table VI. Therefore, the best-fit $g$ PDF, updated by the $p_{T}^{t}$ or $m_{t \bar{t}}$ distribution, does not need to move far from their original position to have a good fit to the data. Another feature we observed from Table VI is that the impact of the $t \bar{t}$ data to $g$ PDF is consistent with the jet data included in the CT14HERA2 fit such that the $\chi^{2} / N$ values updated from CT14HERA2mJ are larger than those from CT14HERA2.

\section{B. ATLAS $7 \mathrm{TeV} W Z$ data}

After observing the constraints of new LHC jet data [1] and $t \bar{t}$ data on the $g$ PDFs, we would also like to see how new LHC Drell-Yan data could modify the quark PDFs. The low-luminosity $\left(35 \mathrm{pb}^{-1}\right)$ ATLAS $7 \mathrm{TeV} W^{ \pm}$and $Z$ cross section data [6] were included in the CT14HERA2 fit. Since then, ATLAS has published the more precise ATLAS $7 \mathrm{TeV} W Z$ data with an integrated luminosity of $4.6 \mathrm{fb}^{-1}$ [42]. Here, we will study the impact of this more precise data on further constraining the CT14HERA2 PDFs.
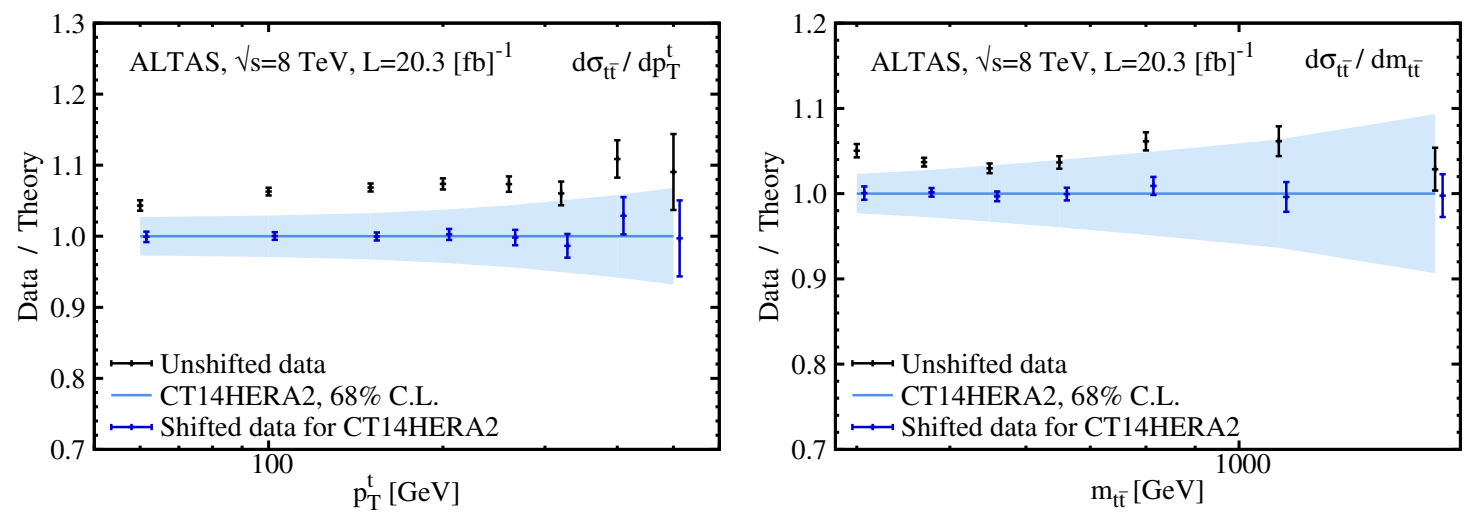

FIG. 21. Data and theory comparison for ATLAS $8 \mathrm{TeV} d \sigma / d p_{T}^{t}$ and $d \sigma / d m_{t \bar{t}}$ data. The uncertainties of theory and data are both at the $68 \%$ C.L. 
TABLE VI. A list of $t \bar{t}$ datasets from CMS [40] and ALTAS [41]. $\chi^{2}$ per data point $\chi^{2} / N$ is shown in the third column for CT14HERA2 and fourth column for CT14HERA2mJ. Both are obtained by adding the $t \bar{t}$ data, one at a time, using ePump.

\begin{tabular}{lccc}
\hline \hline ID & Data & $\chi^{2} / N$ of CT14HERA2 & $\chi^{2} / N$ of CT14HERA2mJ \\
\hline 561 & CMS 8 TeV normalized $d \sigma / \sigma d p_{T}^{t}$ & 4.35 & 5.68 \\
562 & CMS 8 TeV normalized $d \sigma / \sigma d y_{t}$ & 2.63 & 3.06 \\
563 & CMS 8 TeV normalized $d \sigma / \sigma d m_{t \bar{t}}$ & 6.07 & 7.71 \\
564 & CMS 8 TeV normalized $d \sigma / \sigma d y_{t \bar{t}}$ & 2.19 & 3.24 \\
565 & ATLAS 8 TeV absolute $d \sigma / d p_{T}^{t}$ & 0.49 & 0.52 \\
566 & ATLAS 8 TeV absolute $d \sigma / d\left|y_{t}\right|$ & 2.89 & 6.09 \\
567 & ATLAS 8 TeV absolute $d \sigma / d m_{t \bar{t}}$ & 1.19 & 1.00 \\
568 & ATLAS 8 TeV absolute $d \sigma / d\left|y_{t \bar{t}}\right|$ & 5.09 & 9.10 \\
\hline \hline
\end{tabular}
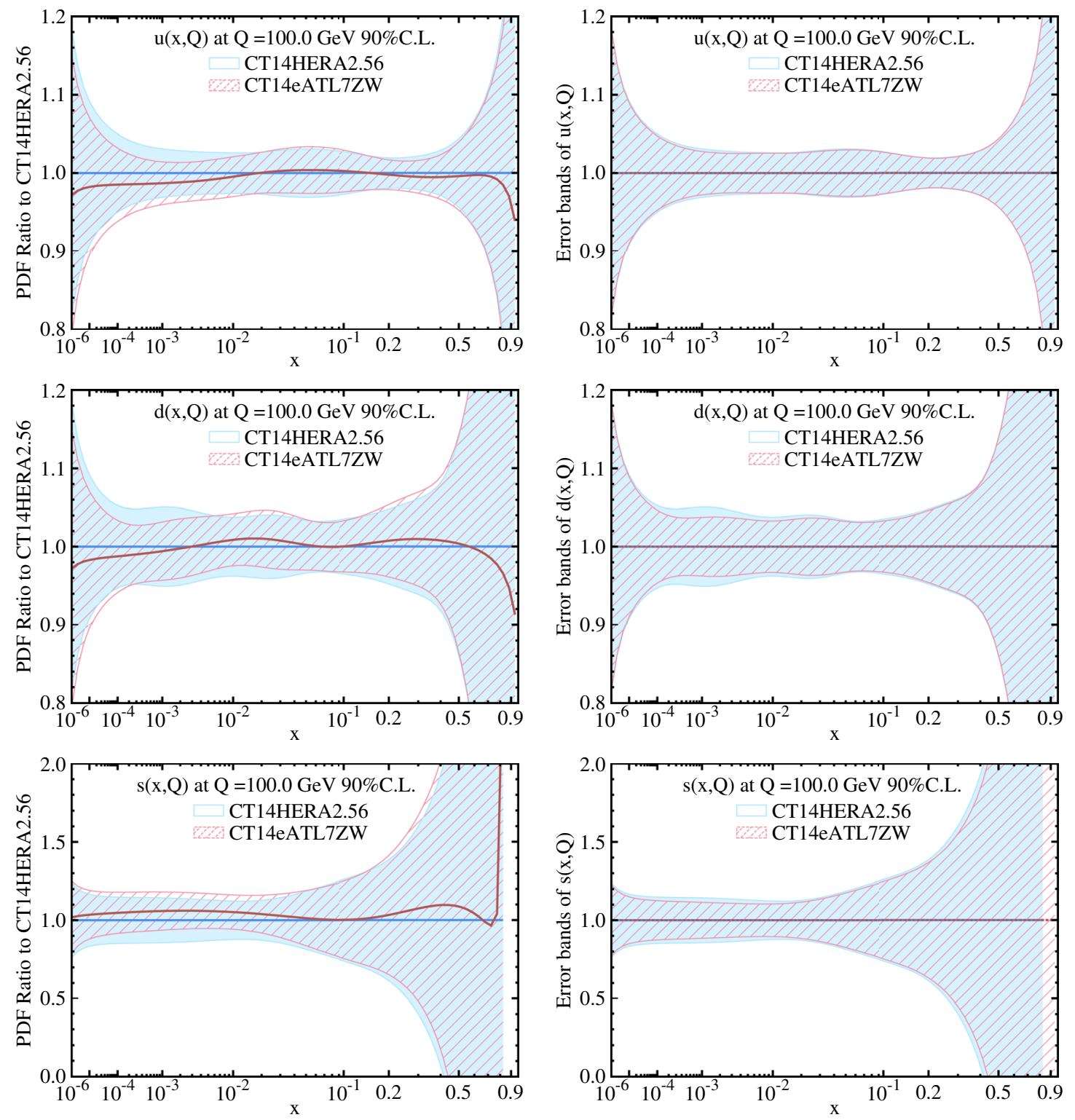

FIG. 22. Updated $u, d$, and $s$ PDFs when the ATLAS 7 TeV $W$ and $Z$ dataset is added to CT14HERA2. The suffix ".56" indicates that here the error bands for CT14HERA2 are computed using 56 eigen-PDFs, including the two gluon extreme sets (labeled as the 55th and 56th eigensets). Left panel: PDF ratios to the CT14HERA2 best fit. Right panel: the error bands relative to their own best fit. 
Strictly speaking, we should first remove the old ATLAS $7 \mathrm{TeV} W Z$ data from the CT14HERA2 global fit and then add the new ATLAS $7 \mathrm{TeV} W Z$ data with ePump, so as not to double-count the ATLAS $7 \mathrm{TeV} W Z$ data contributions. However, since the two datasets are consistent and the new one has about 100 times the integrated luminosity as the old one, the impact of the double-counting should be negligible. Therefore, we shall simply add the new ATLAS $7 \mathrm{TeV} W Z$ data to update the CT14HERA2 PDFs using ePump. Figure 22 shows the updated PDFs. One can see that the new ATLAS $7 \mathrm{TeV} W$ and $Z$ data have a sizable impact on the quark PDFs and their uncertainties, particularly for $x$ ranging from $10^{-4}$ to a few times $10^{-2}$. On the one hand, this is understandable because these data are very precise, with uncertainties less than the percent level. On the other hand, such a large difference between the updated PDFs and the original CT14HERA2 PDFs calls for further investigation.

We note that the new ATLAS $7 \mathrm{TeV} W$ and $Z$ data, to be denoted as "ATL7ZW" data from now on, with a total of 34 data points, cannot be fit well. Its $\chi^{2}$ per data point after the fit by ePump is found to be around 2.7, which is much larger than that found for the full CT14HERA2 global fit (about 1.25) with a total of 3287 data points. Let us consider the two measures, $d^{0}$ and $\tilde{d}^{0}$, introduced in Ref. [1] to assess the quality of the fit given by ePump. Recall that these two measures are the length of the shift in the parameter space of the best-fit PDFs, relative to the original and to the updated $90 \%$ C.L. boundaries, respectively. The ePump output file gives $d^{0}=0.87$ after adding these data, which indicates that the shift of the best-fit parameters nearly touches the $90 \%$ C.L. boundary of the original CT14HERA2 fit. Furthermore, we find a value of $\tilde{d}^{0}=$ 1.49 , which implies that the original best-fit parameter point falls outside of the $90 \%$ C.L. region of the updated fit. This latter result, in particular, suggests that the points in the parameter space used to evaluate the new data may (though not necessarily) be outside the region of validity of the Hessian approximations. Thus, in this case, the result from ePump should be taken with caution, and the shift in the best-fit PDFs found in a true global fit may likely be larger than that given by the ePump program. With that said, we find from ePump updating that adding the ATLAS $7 \mathrm{TeV} W Z$ data to the CT14HERA2 fit would decrease the $u$ and $d$
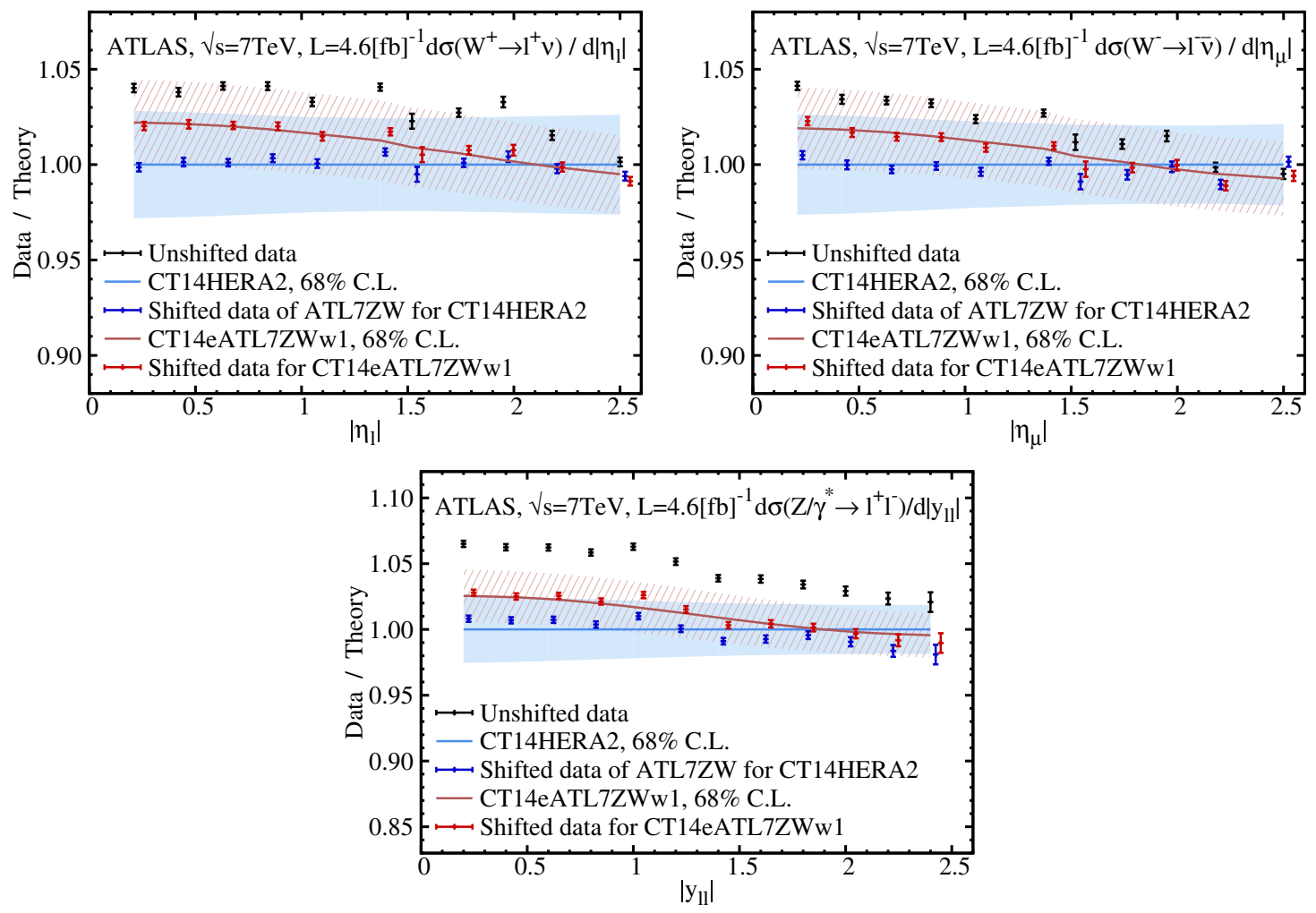

FIG. 23. Comparison between ATLAS $7 \mathrm{TeV} W$ and $Z$ data and the theory predictions for each data point. The raw data are labeled as "unshifted data." The shifted data, after being corrected by the nuisance parameters of the correlated systematic errors, are compared to the theory predictions before (labeled as CT14HERA2, in blue) and after the ATLAS $7 \mathrm{TeV} W$ and $Z$ data is included in the ePump updating with weight of 1 (labeled as CT14eATL7ZWw1, in red). The error bar on each data point includes both statistical and uncorrelated systematic errors, added in quadrature. The error band of theory prediction indicates the PDF induced error at the $68 \%$ C.L. 

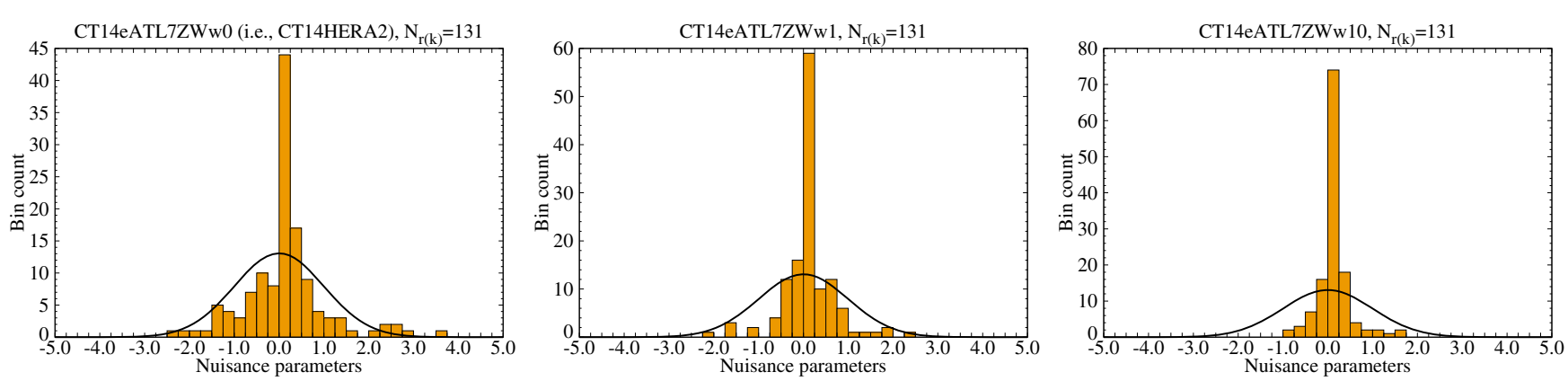

FIG. 24. Distribution of the nuisance parameters for ATLAS $7 \mathrm{TeV} W$ and $Z$ data, after the ePump updating. It shows, from left to right, the results of the ePump-updated fits with the ATLAS $7 \mathrm{TeV} W$ and $Z$ data included with weight of 0 (i.e., CT14HERA2, labeled as CT14eATL7ZWw0), 1 (labeled as CT14eATL7ZWw1), and 10 (labeled as CT14eATL7ZWw10), respectively. The solid curve is the standard normal distribution (with a mean value of 0 and standard deviation of 1) expected in the ideal case.

quark PDFs and increase the $s$ quark PDF at $x=10^{-4} \sim$ $10^{-3}$ and increase the $d$ PDF at $x$ around $10^{-2}$ and 0.3 . Also, the error band of the $d$ PDF is reduced significantly around $x=10^{-3}$, and the error band of the $s$ PDF is reduced for nearly all values of $x$.

In addition to the single-value criteria, one can also compare the data and theory predictions point by point to reveal some more details about the quality of fit, as shown in Fig. 23. First, we find that there is an overall shift for all the raw data points. This means that the correlated systematic errors, weighted by their corresponding nuisance parameters, play an important role in the fitting. Figure 24 shows the distributions of nuisance parameters, before and after updating with ePump. The solid curve in the figure shows a standard normal distribution with a mean value of 0 and standard deviation of 1 . It shows that there are some large nuisance parameters before the updating. Given the large difference between data and theory for CT14HERA2 in Fig. 23, we conclude that ATLAS $7 \mathrm{TeV}$ $W$ and $Z$ data are not described well by the CT14HERA2 PDFs, so we expect a large impact of this dataset to update the CT14HERA2 PDFs. Second, one can see that the ATLAS $7 \mathrm{TeV} W$ and $Z$ data are more precise than the theory predictions, with PDF induced uncertainty included, and even after ePump updating, the precision data still cannot be described well by the theory. This, together with the large contributions from the nuisance parameters, leads to the large $\chi^{2}$ for this dataset.

Given the above discussion, we might expect some tension between the new ATLAS $7 \mathrm{TeV} W$ and $Z$ data and the old datasets included in the CT14HERA2 fit. To examine this, we increase the weight of the ATLAS $7 \mathrm{TeV}$ $W Z$ data while updating the CT14HERA2 PDFs using the ePump program. We can simultaneously obtain the updated predictions for all of the other CT14HERA2 datasets, by including them in ePump as new data, but with zero weight. In this fashion, we can see how the fit to the original datasets changes as the new data are added, in order to investigate for possible tensions. Increasing the weight of the ATLAS $W Z$ data forces ePump to fit this data better; however, if some of the original CT14HERA2 datasets have tension with the $W Z$ data, they will be fitted worse as the weight of this $W Z$ data increases. As discussed in Ref. [43], the goodness of fit to individual dataset can be quantified by the variable "spartyness" $S_{n}$, an equivalent Gaussian variable. A well-fitted dataset should have $S_{n}$ between -1 and 1. An $S_{n}$ smaller than -1 means the dataset is fitted "too" well, and an $S_{n}$ larger than 1 indicates poor fitting.

We find that most of the datasets in CT14HERA2 do not show appreciable tension with the ATLAS $W Z$ data. However, some datasets do exhibit tension, as shown in Fig. 25, which displays the change of spartyness $S_{n}$ for these affected datasets as the weight of the ATLAS $W Z$ data is increased from 0 to 10 . Some of these datasets were not fitted well before (weight equal to 0 ) and become worse as the weight is increased, e.g., the CDF Run- $2 Z$ rapidity data. Others of these datasets were fitted well before but become poorly fitted after the weight is increased, the most significant ones being the NuTeV $\bar{\nu} \mu \mu$ SIDIS, the

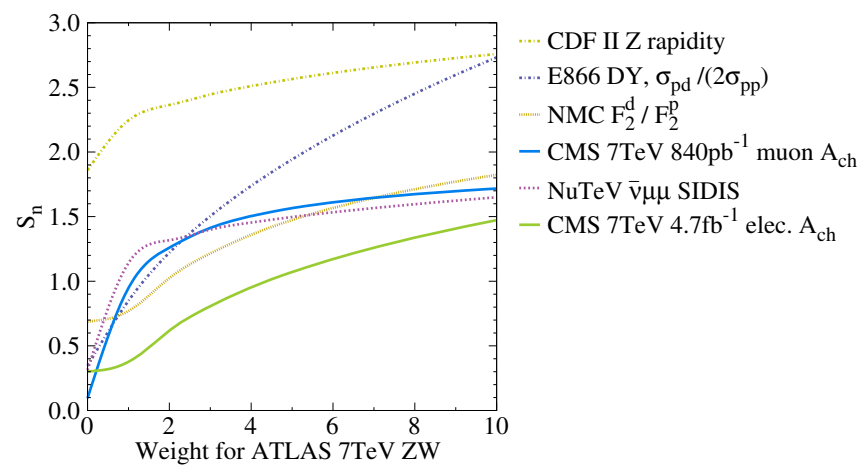

FIG. 25. The change of spartyness $S_{n}$ for some datasets in CT14HERA2 as the weight of ATLAS $7 \mathrm{TeV} W Z$ data is increased from 0 to 10 . Only the datasets with a large change in $S_{n}$ are shown. Note that a weight of zero corresponds to CT14HERA2 fit. 

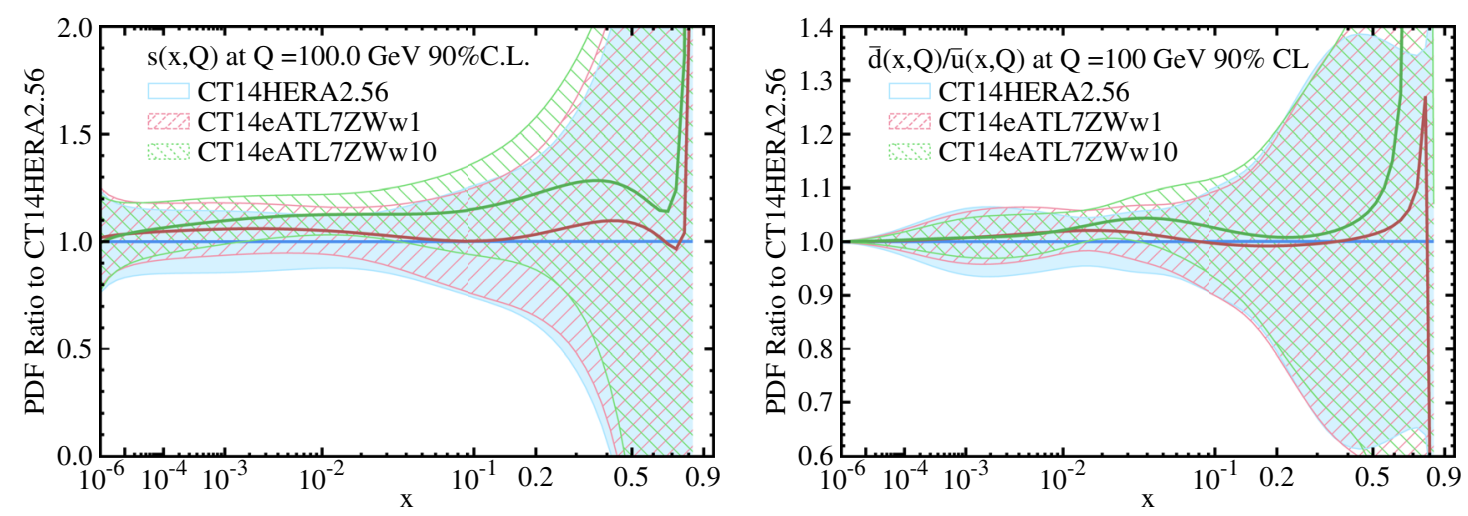

FIG. 26. The updated $s$ PDF and the $\bar{d} / \bar{u}$ PDF ratio, at $Q=100 \mathrm{GeV}$, after ATLAS $7 \mathrm{TeV} W Z$ data are added to CT14HERA2 using ePump, with weights of 1 and 10. As in Fig. 22, the CT14HERA2 error bands are computed using 56 eigen-PDFs.

E866 $\sigma_{p d} /\left(2 \sigma_{p p}\right)$ datasets, and the CMS $7 \mathrm{TeV} \mu$ and electron asymmetry data.

As discussed in Sec. III C, the $s$ PDF is mainly constrained by the (anti)neutrino DIS charged current dimuon data, cf. Fig. 16, and the NuTeV $\bar{\nu} \mu \mu$ SIDIS data impose the strongest constraint on the $s$ PDF among those four datasets. Figure 26 shows the ePump-updated $s$ PDF with weights of 1 and 10 on the ATLAS $W Z$ data. We see that
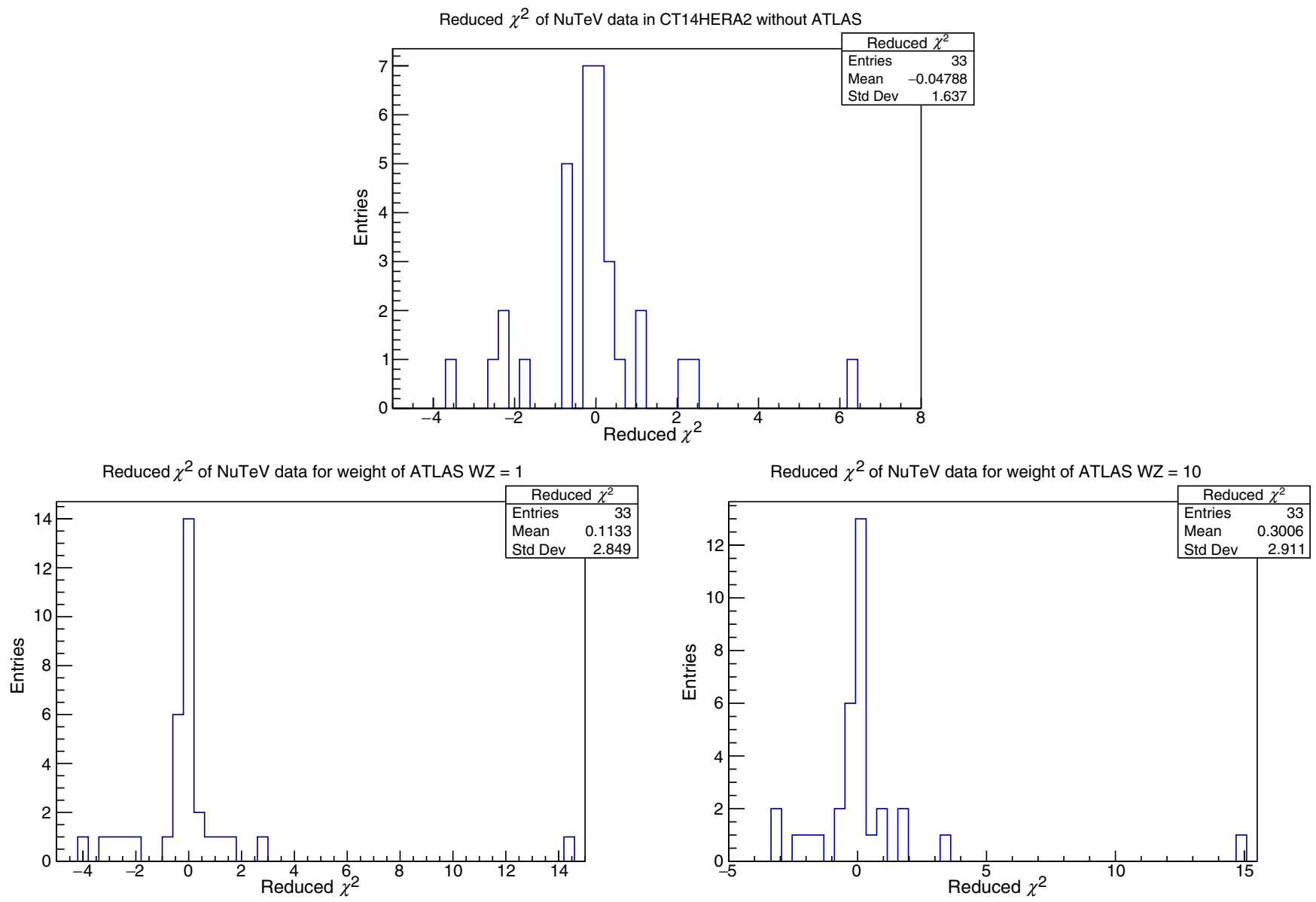

FIG. 27. The distribution of reduced $\chi^{2}$ for the $\mathrm{NuTeV} \bar{\nu} \mu \mu$ data. The upper one is the reduced $\chi^{2}$ in CT14HERA2 before ePump updating. The lower two are the distribution of reduced $\chi^{2}$ when the ATLAS $7 \mathrm{TeV} W$ and $Z$ dataset is included using ePump, with weights equal to 1 and 10, respectively. We see that with the NuTeV $\bar{\nu} \mu \mu$ data included, one data point shows dramatically increasing reduced $\chi^{2}$. In Fig. 28, it is recognized to be the data point with $x=0.015$ and $y=0.776$. When the weight increases from 1 to 10 , their features do not change largely. Std Dev—standard deviation. 


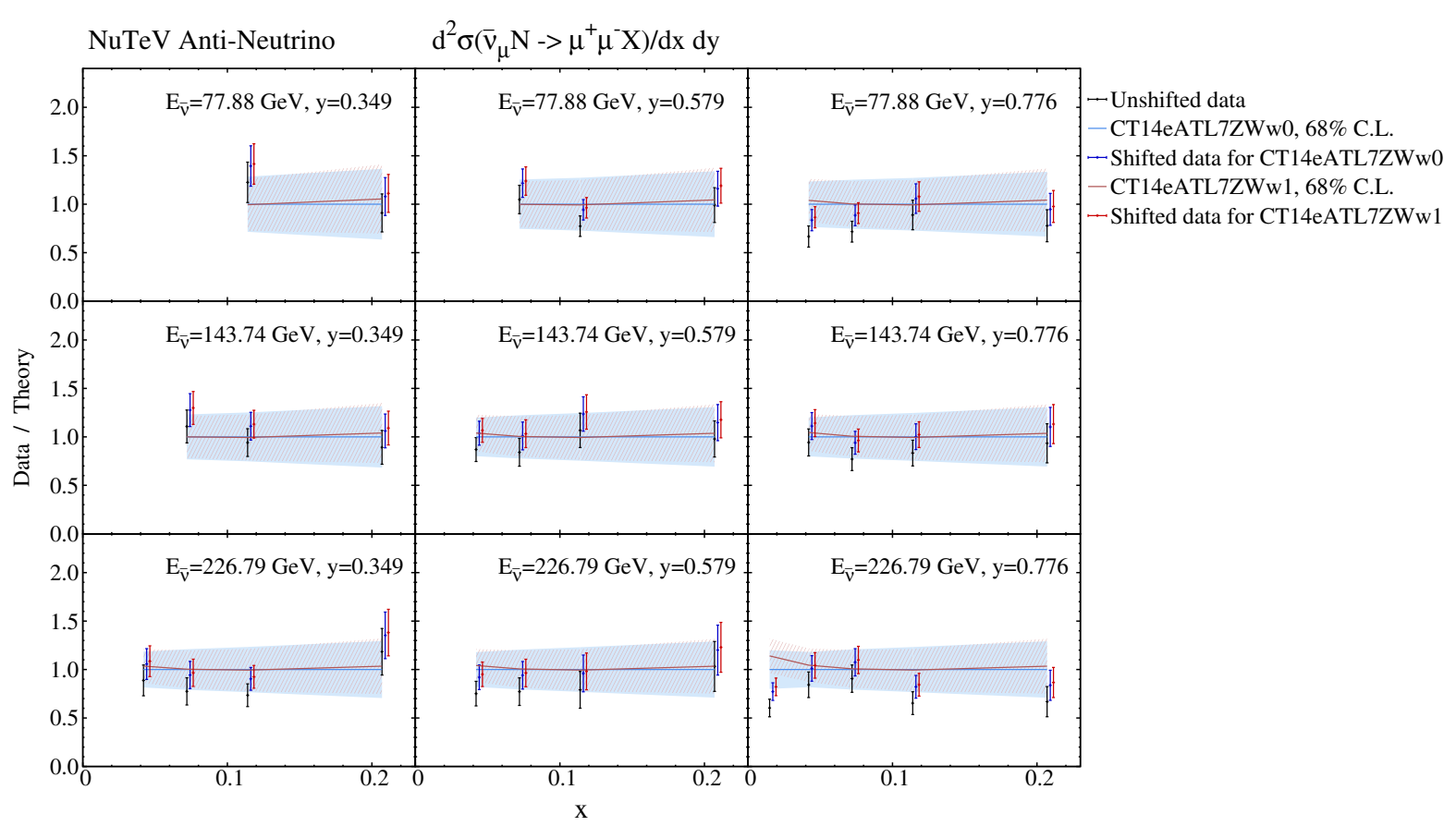

FIG. 28. Comparison between $\mathrm{NuTeV} \bar{\nu} \mu \mu$ data and the theory predictions for each data point, before (labeled as CT14eATL7ZWw0) and after (labeled as CT14eATL7ZWw1) ATLAS $7 \mathrm{TeV}$ WZ data is included to update the CT14HERA2 PDFs. The unshifted data are shown at their original $x$ values and the shifted data are shown at slightly shifted $x$ values for clear comparison. From the bottom to the right figure we can identify the data point with $x=0.015$ and $y=0.776$ as the one possessing tension with the ATLAS $7 \mathrm{TeV} W Z$ data.

the ATLAS $W Z$ data prefer larger values of the $s$ PDF, while the NuTeV data prefer smaller values at $x$ around a few times $10^{-2}$. When the weight of the ATLAS $7 \mathrm{TeV} W$ and $Z$ data increases, the reduced $\chi^{2}$ of the $\mathrm{NuTeV}$ data point with $x=0.015$ and $y=0.776$ increases dramatically, as shown in Figs. 27 and 28, which indicates the tension between the ATLAS $7 \mathrm{TeV} W Z$ data and the $\mathrm{NuTeV}$ data.

In Fig. 26, we also show the updated $\bar{d} / \bar{u}$ PDF ratio plot with weights of 1 and 10 on the ATLAS $W Z$ data. We find that the ATLAS $W Z$ data prefer a larger value of $\bar{d} / \bar{u}$ ratio at $x$ around $10^{-3}$ to $10^{-1}$. This is to be compared with what we concluded in Sec. III, that the E866 $\sigma_{p d} /\left(2 \sigma_{p p}\right)$ dataset is crucial for constraining $\bar{d} / \bar{u}$ and $d_{v} / u_{v}$ at $x$ around $10^{-2}$ to 0.2 , cf. Fig. 12. Therefore, increasing the weight of ATLAS $7 \mathrm{TeV} W Z$ data contradicts the fit of E866 data and leads to the tension. This can also be illustrated by the comparison between data and theory before and after ATLAS $7 \mathrm{TeV} W Z$ dataset is included, see Fig. 29, in which we find a deviation of the theory predictions from the data for large rapidity when the weight of ATLAS $7 \mathrm{TeV} W Z$ data is increased from 1 to 10 .

For the CMS asymmetry data, the tension with the ATLAS $W Z$ data can be demonstrated in the same way, by comparing theory with data for each data point, as the weight of ATLAS $7 \mathrm{TeV}$ WZ data is increased. In Figs. 30, the comparison is shown for CMS $\mu$ and electron asymmetry data. It is apparent that as the weight of ATLAS $7 \mathrm{TeV} W Z$ data is increased from 1 to 10 both the theory predictions of CMS $\mu$ and electron asymmetry have an overall upward shift compared to the data for almost all of the data points. Given the precision of the CMS data, this leads to a large $\chi^{2}$, which is reflected by the rapid increase of spartyness in Fig. 25.

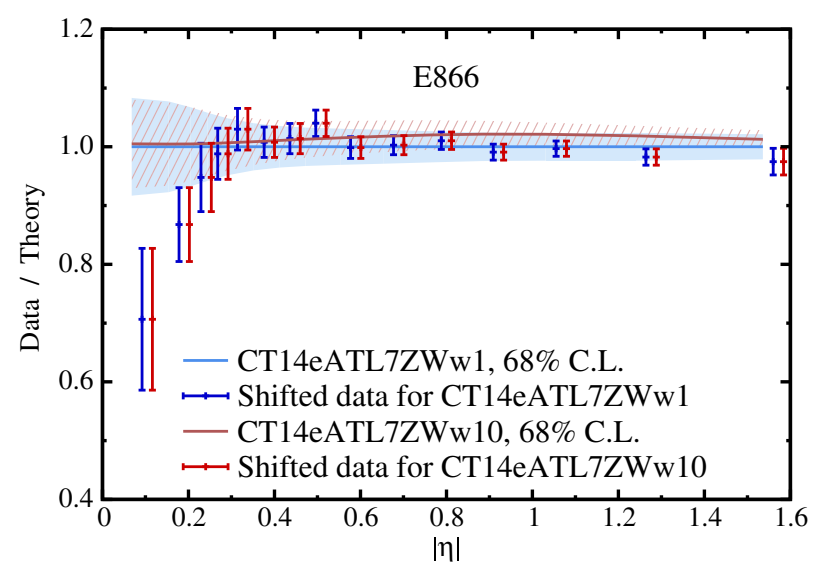

FIG. 29. Comparison between E866 data and the theory predictions for each data point, as the weight of ATLAS $7 \mathrm{TeV} W Z$ data is increased from 1 to 10 . 

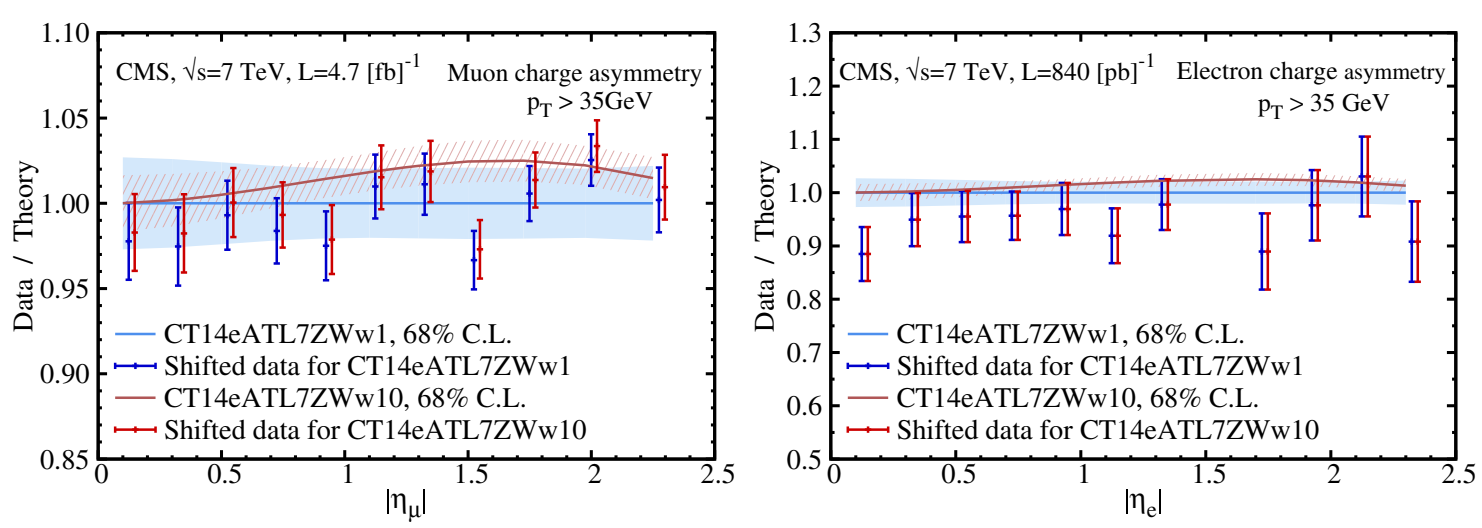

FIG. 30. Comparison between $\mathrm{CMS} 7 \mathrm{TeV} \mu$ and electron asymmetry data and the theory predictions for each data point, as the weight of ATLAS $7 \mathrm{TeV} W Z$ data is increased from 1 to 10 .

\section{CONCLUSIONS}

A fast and efficient tool for estimating the impact of new data on the PDFs is essential in this high-precision era of the LHC. In this paper, we have tested just such a tool, ePump, both as to its effectiveness and its validity.

We have validated ePump in three trials, where we started with a base PDF set obtained from a global fit with some subset of the CT14HERA2 data removed. We used ePump to update these base PDFs with the missing datasets and then compared with the CT14HERA2 PDFs. In all three trials (updating with DIS, Drell-Yan, or jet datasets) the ePump results are very close to the CT14HERA2 global-fit results. This is important because the goal is to have the best approximation to the full global fit as possible. Of course, there are some differences, but they are either small compared to the error bands or happen in very small- or large- $x$ regions, where the PDFs depend strongly on the parametrization forms. Another case in which the ePump approximations break down is when there are strong tensions between the new and old data. As we have seen, the global fit may increase the error bands, but this will never happen with ePump. Again, we emphasize that ePump is not meant to replace the global fit. However, even in situations with tensions between new and old data, it still gives qualitatively correct results and therefore provides a useful tool for judging the impact of new data. In addition to updating PDFs, ePump can also update the observables at the same time without the need for recalculating. An example of this use of ePump was given for the predictions of $\sigma(g g \rightarrow h)$.

A big advantage of ePump is that it can run very fast. This was exploited to study the impact of different datasets in the CT14HERA2 fit. Summaries of the impact of each of the datasets were given in Tables II-IV. The impact of each dataset is strongest for some particular flavors and for dataset's relevant region of $x$. But it also depends on the precision of the data and their agreement with the current PDFs. Therefore, even for two datasets that are sensitive to the same kinematic range and flavor content, they do not necessarily have the same effect on the PDFs. One remarkable thing we found is that among the 33 datasets in CT14HERA2, only one jet, five Drell-Yan, and eight DIS datasets ${ }^{3}$ have the dominant effects. Just by including these datasets, we can reproduce the bulk part of the CT14HERA2 fit. The other datasets are only responsible for some fine structures of the PDFs.

It is incredible that we can fit thousands of data points with only 27 or 28 parameters. This triumph strongly shows the effectiveness of the QCD improved parton model. In such an era of precision, it has become an important and indispensable task to reduce the uncertainties of PDFs. So, the natural question is as follows: what kinds of observables can reduce the PDF uncertainties? The purpose of ePump is to help answer this question. The new data to be investigated by ePump can be new experimental data, or it can be simulated pseudodata, the impact of which one might be interested to see. An example of this second scenario was presented in Ref. [5], in which ePump was used to show that with increased precision and an optimal choice of kinematic variables the high-invariant mass Drell-Yan processes can greatly reduce the PDF uncertainties. In this paper, we examined the impact of the latest $t \bar{t}$ data and $W$ and $Z$ data at the LHC on the CT14HERA2 PDFs. We found that the $t \bar{t}$ data have the potential to reduce $g$ PDF uncertainties given increased luminosity and that the highprecision $W$ and $Z$ data also can provide strong constraints on the quark PDFs. Of course, these results will be refined quantitatively by a full global fit, but ePump can quickly assess the qualitative. Similar studies can also be done for other processes. We expect ePump to play an important role in the study of PDFs and to assist in the understanding and reduction of theoretical errors in the current era of the highluminosity LHC. The complete ePump package, together with detailed instructions for installing and file formatting and additional output files relevant to this study, can be found at the website http://hep.pa.msu.edu/epump/.

\footnotetext{
${ }^{3}$ This includes HERA I + II data as well.
} 
Before closing this section, we would like to make two additional remarks. First, we note that the comparison made in this paper between the ePumpand global-fit analyses holds at any given fixed order, either next-to-leading order or nextto-next-to-leading order, of theory calculations. Second, we note that the default XFITTER profiling analysis [4] can be reproduced by ePump, but using a global tolerance set to 1 . However, as discussed in the Appendix, setting tolerance to be 1 will greatly overestimate the impact of a given new dataset when updating the existing PDFs in the CTEQ-TEA (CT) PDF global analysis framework.

\section{ACKNOWLEDGMENTS}

We thank our CTEQ-TEA colleagues for support and discussions. This work was supported by the U.S. National Science Foundation under Grant No. PHY-1719914. C.-P. Y. is also grateful for the support from the Wu-Ki Tung endowed chair in particle physics. The work of S. D. was supported by the National Natural Science Foundation of China under Grant No. 11965020.

\section{APPENDIX: THE ROLE OF THE TOLERANCE $T$ IN UPDATING OF PDFs}

When constructing Hessian eigenvector PDFs for error estimation, different PDF groups have made different choices for defining the PDF errors. This includes the choice of $68 \%$ vs $90 \%$ C.L., the inclusion of a global tolerance $T$, or the imposition of dynamical tolerances $T_{i}^{ \pm}$ along different eigenvector directions. This has caused some confusion when the eigenvector PDFs are then used in the updating of PDFs with new data. We shall clarify this issue here in the context of ePump.

The basic premise of the Hessian approximation is that, near its global minimum, $\chi^{2}$ can be written as a quadratic function of the PDF parameters $\left\{z_{i} ; i=1, N\right\}$. For the CT14HERA2 fit, $N=28$ or 27, depending on whether the gluon extreme sets are included or not. By shifting, rotating, and rescaling the PDF parameters, we can write $\Delta \chi^{2}$ for the original datasets as

$$
\Delta \chi_{\text {old }}^{2}=T^{2} \sum_{i=1}^{N} z_{i}^{2}
$$

The parameters $z_{i}$ have been chosen here so that $z_{i}=0$ (for all $i$ ) for the best-fit PDF set $f^{0}$, while $z_{i}= \pm \delta_{i j}$ for the $2 N$ eigenvector PDFs $f^{ \pm j}$. In this way, each of the eigenvector PDF sets corresponds to a $\Delta \chi_{\text {old }}^{2}=T^{2}$, where $T$ is an overall global tolerance parameter. If the various datasets were all internally consistent and satisfied Gaussian statistics, then one should use $T=1$ at the $68 \%$ C.L. or $T=$ 1.645 at the $90 \%$ C.L. However, because of inconsistencies between the various datasets, as well as uncertainties arising from the initial choice of PDF parametrization forms, the CTEQ-TEA group has historically chosen a larger value of $T=10$ at the $90 \%$ C.L.

Another variation in defining the Hessian PDF errors is in the imposition of dynamical tolerances, which have been used in recent CTEQ-TEA [7,9] and MMHT [44] PDF sets. The idea here is that if the constraints on a given PDF eigenvector direction come dominantly from a single dataset (or several self-consistent sets) then the overall global tolerance produces too large of a PDF error. By incorporating separate constraints from individual experiments, one determines that a $\Delta \chi_{\text {old }}^{2}=\left(T_{i}^{ \pm}\right)^{2}<T^{2}$ in the particular eigenvector direction should correspond to the given C.L. Keeping Eq. (A1) unchanged, this implies that the Hessian eigenvector PDFs now must correspond to $z_{i}= \pm\left(T_{j}^{ \pm} / T\right) \delta_{i j}$. (It can easily be seen that in the presence of dynamical tolerances the global parameter $T$ scales out of all calculated observables.)

We emphasize here that, although the different choices of tolerance parameters (including whether global or dynamical), as well as the choice of $68 \%$ or $90 \%$ C.L, give different results and/or interpretations for the PDF errors, they all give a self-consistent description of $\chi_{\text {old }}^{2}$ around the global minimum.

The next step in updating the PDFs in the Hessian approach is to add the contribution of the new dataset (or sets) to $\chi^{2}$, yielding

$\chi_{\text {new }}^{2}=T^{2} \sum_{i=1}^{N} z_{i}^{2}+w \sum_{\alpha, \beta=1}^{N_{X}}\left(X_{\alpha}^{E}-X_{\alpha}(\mathbf{z})\right) C_{\alpha \beta}^{-1}\left(X_{\beta}^{E}-X_{\beta}(\mathbf{z})\right)$,

where $N_{X}$ is the number of new data points, $X_{\alpha}^{E}$ are the experimental data values, $X_{\alpha}(\mathbf{z})$ are the theoretical predictions, and $C_{\alpha \beta}^{-1}$ is the experimental inverse covariance matrix. We have also included a weight factor $w$ that may be assigned to the new set of data, which by default is set to be 1. To linear order in the PDF parameters (assuming that a global tolerance is used), we can express the theoretical predictions as

$$
X_{\alpha}(\mathbf{z})=X_{\alpha}(0)+\sum_{i=1}^{N}\left(\frac{X_{\alpha}^{+i}-X_{\alpha}^{-i}}{2}\right) z_{i},
$$

where $X_{\alpha}^{ \pm i}$ is the theoretical prediction of $X_{\alpha}$ calculated with the error PDFs $f^{ \pm i}$. We emphasize that Eq. (A3) is valid only when $z_{i}= \pm \delta_{i j}$ corresponds to the error PDFs $f^{ \pm j}$. At this stage, $\chi_{\text {new }}^{2}$ can now be minimized to obtain the new value of the best-fit parameters, which can be used to obtain updated best-fit PDFs. The generalization of Eq. (A3) for dynamical tolerances and the extension of this equation to include diagonal quadratic terms are given in Ref. [1] and are implemented in ePump. In addition, ePump produces an updated set of error PDFs, under the same 

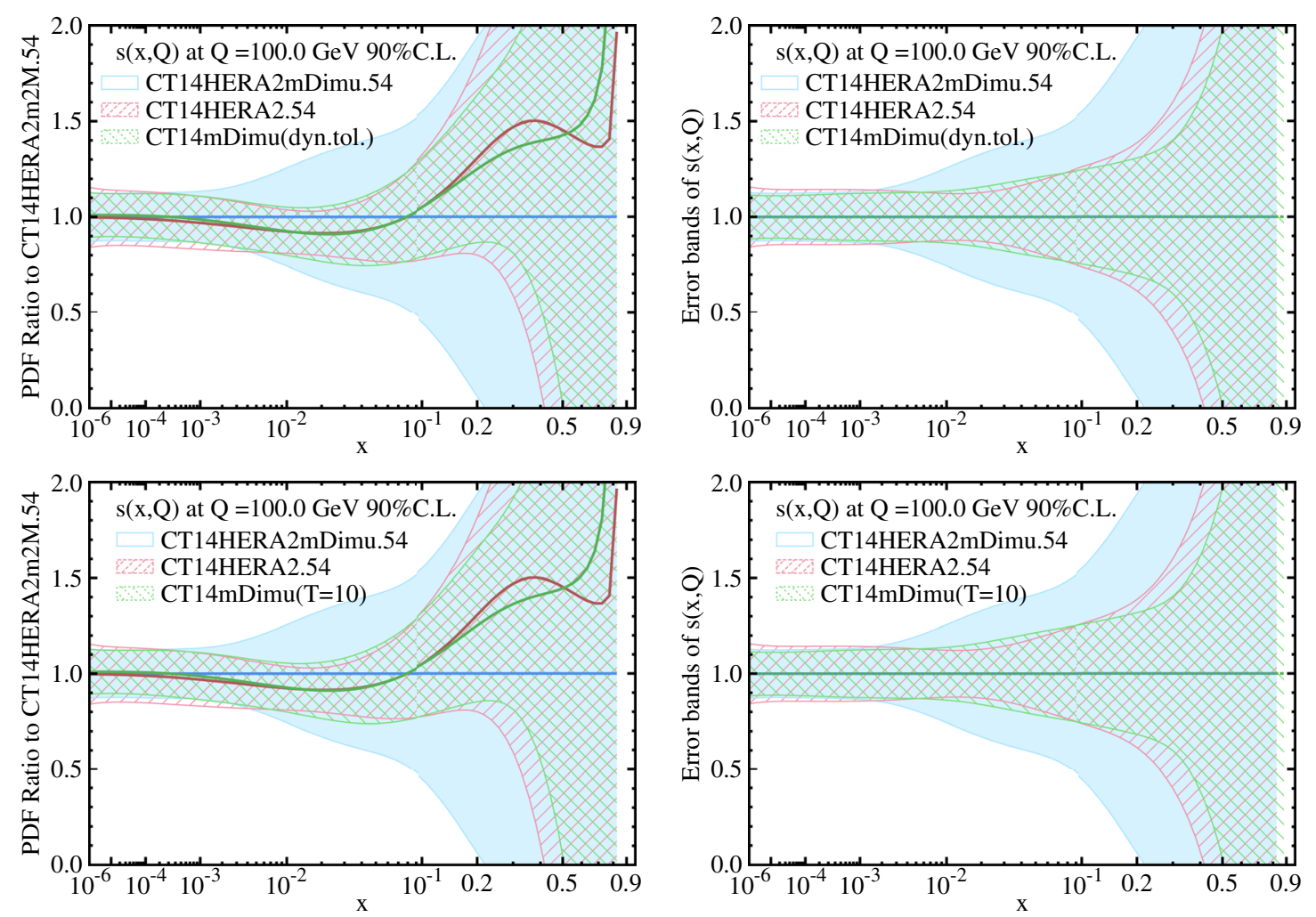

FIG. 31. Comparison of ePump-updated $s$ PDFs with the global-fit result, at $Q=100 \mathrm{GeV}$. In the upper two plots, dynamical tolerance was used [labeled as CT14mDimu(dyn.tol.)]. In the lower two plots, dynamical tolerance was turned off, and $T^{2}=100$ was assigned [labeled as CT14mDimu $(T=10)$ ]. These two results are very similar, and both reproduce CT14HERA2 with good agreement. Left panel: the PDF ratios over the best fit of the base CT14HERA2mD. Right panel: the error bands relative to their own best fit.

tolerance and confidence-level assumptions of the original error PDFs.

Although these results were given previously in Ref. [1], our purpose for restating them here is to make it clear that the choice of the global tolerance $T$ or the use of dynamical tolerances in the updating of the PDFs should not be chosen freely but rather is determined by the original Hessian error PDF sets. In particular, if the error PDFs were determined using a global tolerance of $T=10$, then the use of Eq. (A3) is only consistent if the value $T=10$ is used in Eq. (A2). It is straightforward to show that if the tolerance were set to $T=1$ in Eq. (A2) while using such error PDFs it is equivalent to weighting the new data by a factor $w=100$ in the update.

We exemplify this by performing the following exercise. As demonstrated in Sec. III C, the dimuon data are almost entirely responsible for constraining the $s$ PDF. Therefore, we remove the dimuon data from the CT14HERA2 datasets and perform a new global fit, named CT14HERA2mDimu. Then, we use ePump to add back the dimuon data. If everything works perfectly, we should expect that the ePump-updated $s$ PDF will agree with CT14HERA2. Also, based on the present discussion, we should perform the update using the dynamical tolerances, but we shall also try other choices for the tolerance to see the results.
We first compare the update using dynamical tolerances with that using a global tolerance of $T^{2}=100$. The results are shown in Fig. 31. In this case, we find that these two updates give very similar predictions and reproduce CT14HERA2 very well. On checking the dynamical tolerance values for CT14HERA2mDimu, we discovered that the eigenvector directions that are most sensitive to the dimuon data have $\left(T_{i}^{ \pm}\right)^{2} \simeq 90$, which explains why the update is not much different when using a global $T^{2}=100$.

Next, we display the results using global tolerance squares of $T^{2}=1$ and $T^{2}=1.645^{2}=2.706$. These correspond to the naive use of Gaussian statistics at the $68 \%$ and $90 \%$ C.L.s, respectively, but are inconsistent with the error PDFs used in this update. As explained above, using these small values of $T^{2}$ with the given error PDFs is equivalent to overweighting these data by a large value. The $s$ PDFs after these updates are shown in Fig. 32. Interestingly, the agreement of the best-fit updates is not too bad when compared with CT14HERA2 (though not as good as when using dynamical tolerances). This can be understood by the fact that the strange quark PDF is mostly determined by the dimuon data, so overweighting these data does not shift the central value by much. However, one can see that the $s$ PDF error bands for these two values of the tolerance are much smaller than that of CT14HERA2, 

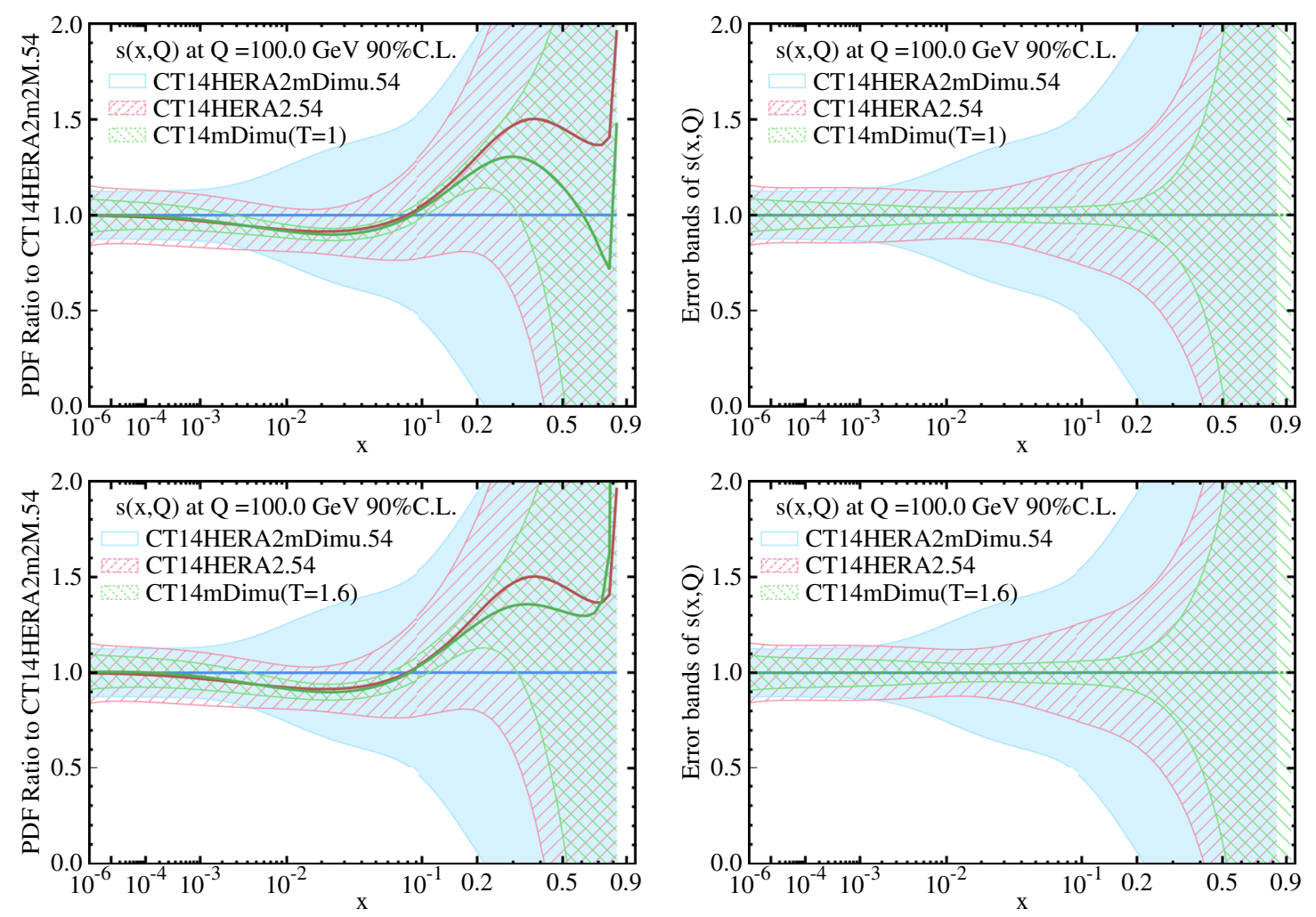

FIG. 32. Comparison of ePump-updated $s$ PDFs with the global-fit result, at $Q=100 \mathrm{GeV}$. In the upper two plots, $T^{2}=1$ was used [labeled as CT14mDimu $(T=1)$ ], while in the lower two plots, and $T^{2}=1.645^{2}=2.706$ was assigned [labeled as CT14mDimu $\left.(T=1)\right]$. Dynamical tolerance was turned off in both cases. These two results cannot reproduce CT14HERA2, giving too-small error bands. Left panel: the PDF ratios over the best fit of the base CT14HERA2m2M. Right panel: the error bands relative to their own best fit.

so using $T^{2}=1$ or $T^{2}=2.706$ would greatly overestimate the effect of these data on reducing the PDF errors.

In conclusion, to best reproduce CT14HERA2 global fit, one should use dynamical tolerance in ePump. Setting tolerance to be 1 will greatly overestimate the impact of a given new dataset when updating the existing PDFs in the CT PDF global analysis framework. This conclusion also holds for using MMHT2014 [44] and PDF4LHC15 [45] PDFs in profiling analysis to study the impact of a new (pseudo)data on updating the existing PDFs.
[1] C. Schmidt, J. Pumplin, and C. P. Yuan, Phys. Rev. D 98, 094005 (2018).

[2] H. Paukkunen and P. Zurita, J. High Energy Phys. 12 (2014) 100.

[3] H. Paukkunen and C. A. Salgado, Phys. Rev. Lett. 110, 212301 (2013).

[4] S. Camarda et al. (HERAFitter developers' Team), Eur. Phys. J. C 75, 458 (2015).

[5] C. Willis, R. Brock, D. Hayden, T. J. Hou, J. Isaacson, C. Schmidt, and C. P. Yuan, Phys. Rev. D 99, 054004 (2019).

[6] G. Aad et al. (ATLAS Collaboration), Phys. Rev. D 85, 072004 (2012).

[7] T. J. Hou, S. Dulat, J. Gao, M. Guzzi, J. Huston, P. Nadolsky, J. Pumplin, C. Schmidt, D. Stump, and C.-P. Yuan, Phys. Rev. D 95, 034003 (2017).
[8] H. Abramowicz et al. (H1 and ZEUS Collaborations), Eur. Phys. J. C 75, 580 (2015).

[9] S. Dulat, T.-J. Hou, J. Gao, M. Guzzi, J. Huston, P. Nadolsky, J. Pumplin, C. Schmidt, D. Stump, and C.-P. Yuan, Phys. Rev. D 93, 033006 (2016).

[10] T. Aaltonen et al. (CDF Collaboration), Phys. Rev. D 78, 052006 (2008); 79, 119902(E) (2009).

[11] V. M. Abazov et al. (D0 Collaboration), Phys. Rev. Lett. 101, 062001 (2008).

[12] G. Aad et al. (ATLAS Collaboration), Phys. Rev. D 86, 014022 (2012).

[13] S. Chatrchyan et al. (CMS Collaboration), Phys. Rev. D 87, 112002 (2013); 87, 119902(E) (2013).

[14] S. Chatrchyan et al. (CMS Collaboration), Phys. Rev. D 90 , 032004 (2014). 
[15] S. Chatrchyan et al. (CMS Collaboration), Phys. Rev. Lett. 109, 111806 (2012).

[16] V. M. Abazov et al. (D0 Collaboration), Phys. Rev. D 77, 011106 (2008).

[17] R. S. Towell et al. (NuSea Collaboration), Phys. Rev. D 64, 052002 (2001).

[18] D. A. Mason, https://doi.org/10.2172/879078 (2006).

[19] M. Goncharov et al. (NuTeV Collaboration), Phys. Rev. D 64, 112006 (2001).

[20] U. K. Yang et al. (CCFR/NuTeV Collaboration), Phys. Rev. Lett. 86, 2742 (2001).

[21] W. G. Seligman et al., Phys. Rev. Lett. 79, 1213 (1997).

[22] J. P. Berge et al., Z. Phys. C 49, 187 (1991).

[23] M. Arneodo et al. (New Muon Collaboration), Nucl. Phys. B483, 3 (1997).

[24] A. C. Benvenuti et al. (BCDMS Collaboration), Phys. Lett. B 223, 485 (1989).

[25] A. C. Benvenuti et al. (BCDMS Collaboration), Phys. Lett. B 237, 592 (1990).

[26] http://hep.pa.msu.edu/epump/.

[27] A. Aktas et al. (H1 Collaboration), Eur. Phys. J. C 40, 349 (2005).

[28] H. Abramowicz et al. (H1 and ZEUS Collaborations), Eur. Phys. J. C 73, 2311 (2013).

[29] F. D. Aaron et al. (H1 Collaboration), Eur. Phys. J. C 71, 1579 (2011).

[30] G. Moreno et al., Phys. Rev. D 43, 2815 (1991).

[31] J. C. Webb et al. (FNAL E866/NuSea Collaboration), arXiv: hep-ex/0302019.
[32] F. Abe et al. (CDF Collaboration), Phys. Rev. Lett. 77, 2616 (1996).

[33] D. Acosta et al. (CDF Collaboration), Phys. Rev. D 71, 051104 (2005).

[34] R. Aaij et al. (LHCb Collaboration), J. High Energy Phys. 06 (2012) 058.

[35] V. M. Abazov et al. (D0 Collaboration), Phys. Lett. B 658, 112 (2008).

[36] T. A. Aaltonen et al. (CDF Collaboration), Phys. Lett. B 692, 232 (2010).

[37] V. M. Abazov et al. (D0 Collaboration), Phys. Rev. D 91, 032007 (2015); 91, 079901(E) (2015).

[38] V. Barone, C. Pascaud, and F. Zomer, Eur. Phys. J. C 12, 243 (2000).

[39] V. Khachatryan et al. (CMS Collaboration), J. High Energy Phys. 03 (2017) 156.

[40] V. Khachatryan et al. (CMS Collaboration), Eur. Phys. J. C 75, 542 (2015).

[41] G. Aad et al. (ATLAS Collaboration), Eur. Phys. J. C 76, 538 (2016).

[42] M. Aaboud et al. (ATLAS Collaboration), Eur. Phys. J. C 77, 367 (2017).

[43] S. Dulat, T. J. Hou, J. Gao, J. Huston, J. Pumplin, C. Schmidt, D. Stump, and C.-P. Yuan, Phys. Rev. D 89, 073004 (2014).

[44] L. A. Harland-Lang, A. D. Martin, P. Motylinski, and R. S. Thorne, Eur. Phys. J. C 75, 204 (2015).

[45] J. Butterworth et al., J. Phys. G 43, 023001 (2016). 